\title{
Combined visualisation of cavitation and vortical structures in a real-size optical diesel injector
}

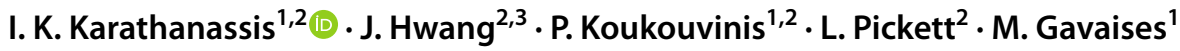

Received: 9 August 2020 / Revised: 17 October 2020 / Accepted: 9 November 2020 / Published online: 23 December 2020

(c) The Author(s) 2020

\begin{abstract}
A high-speed flow visualisation set-up comprising of combined diffuse backlight illumination (DBI) and schlieren imaging has been developed to illustrate the highly transient, two-phase flow arising in a real-size optical fuel injector. The different illumination nature of the two techniques, diffuse and parallel light respectively, allows for the capturing of refractive-index gradients due to the presence of both interfaces and density gradients within the orifice. Hence, the onset of cavitation and secondary-flow motion within the sac and injector hole can be concurrently visualised. Experiments were conducted utilising a diesel injector fitted with a single-hole transparent tip (ECN spray D) at injection pressures of 700-900 bar and ambient pressures in the range of 1-20 bar. High-speed DBI images obtained at 100,000 fps revealed that the orifice, due to its tapered layout, is mildly cavitating with relatively constant cavity sheets arising mainly in regions of manufacturing imperfections. Nevertheless, schlieren images obtained at the same frame rate demonstrated that a multitude of vortices with short lifetimes arise at different scales in the sac and nozzle regions during the entire duration of the injection cycle but the vortices do not necessarily result in phase change. The magnitude and exact location of coherent vortical structures have a measurable influence on the dynamics of the spray emerging downstream the injector outlet, leading to distinct differences in the variation of its cone angle depending on the injection and ambient pressures examined.
\end{abstract}

Electronic supplementary material The online version of this article (https://doi.org/10.1007/s00348-020-03096-1) contains supplementary material, which is available to authorized users.

I. K. Karathanassis

ioannis.karathanassis@city.ac.uk

1 School of Mathematics, Computer Science and Engineering, City, University of London, Northampton Square,

London EC1V 0HB, UK

2 Combustion Research Facility, Sandia National Laboratories, 7011 East Avenue, Livermore, CA 94550, USA

3 Present Address: Department of Mechanical Engineering, Centre for Advanced Vehicular Systems (CAVS), Mississippi State University, Starkville, MS 39762, USA 


\section{Graphic abstract}
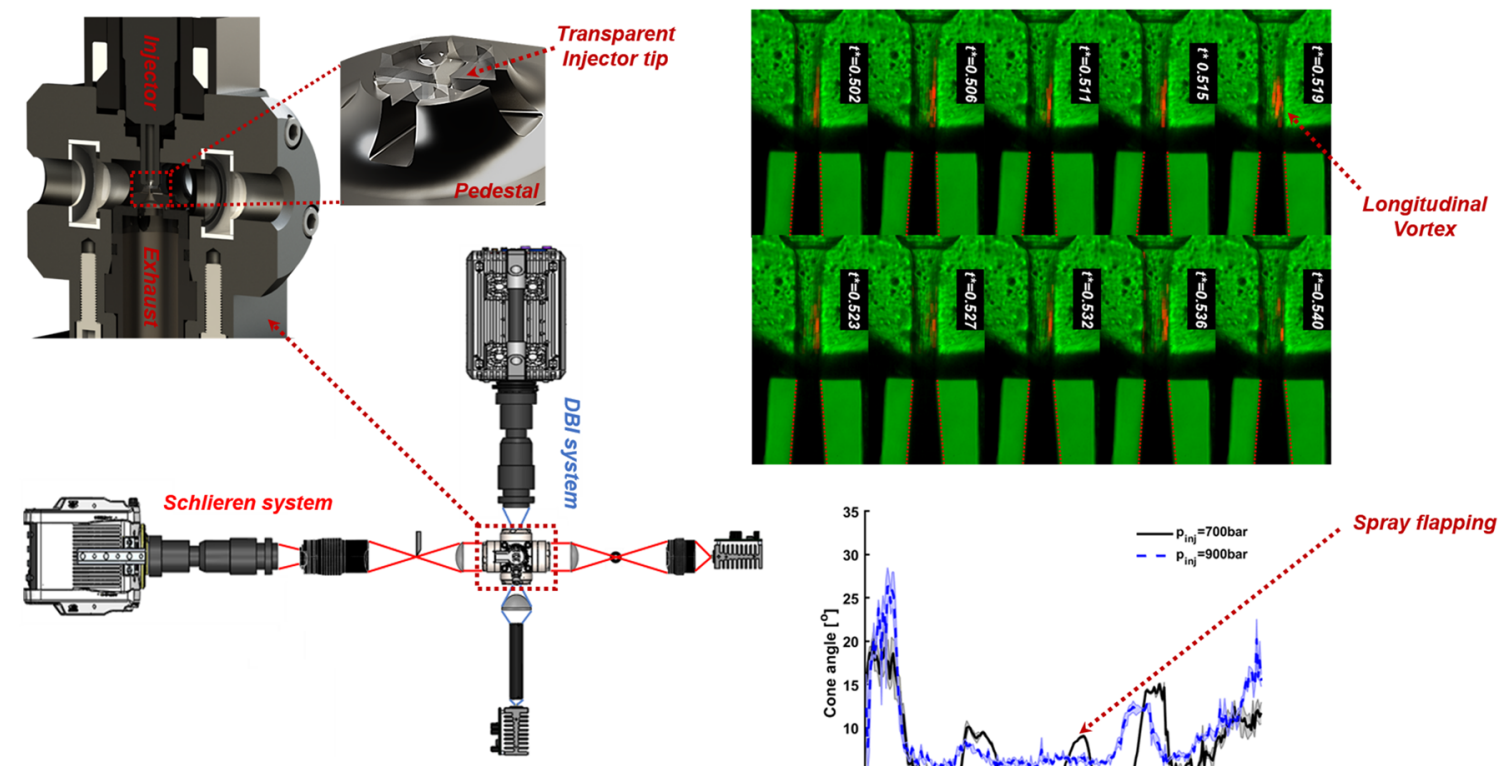

Concurrent DBI/schlieren visualisation of transparent diesel injector

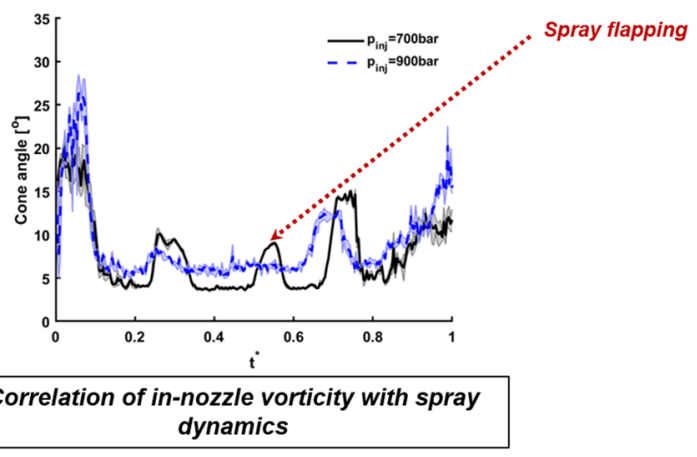

\section{Introduction}

Intense technological efforts and legislation incentives currently in place dictate the imminent electrification of passenger cars and light-duty vehicles incorporating gasoline IC engines. Recent forecasts are suggesting that electric passenger cars will achieve a market penetration of almost $58 \%$ by 2040 (Bloomberg 2020). Nevertheless, at least for the next 3 decades, heavy-duty vehicles will be primarily powered by diesel engines, as current state-of-the-art limitations, for instance battery capacity and thermal management, do not allow a similar technology shift when high-power output for an extended period of time is required. It becomes therefore evident that improvement of the performance and environmental efficiency of modern diesel engines is vital from both economical and societal standpoints.

Combustion quality, designating the IC-engine performance and pollutants emissions, is crucially influenced by the characteristics of the fuel spray directly injected into the cylinder and the effectiveness of subsequent mixing with air. Fuel-injection equipment serves a vital role in such fuel delivery and atomisation processes while operating at extreme pressures, over 2000 bar, and under strict performance criteria, e.g. high discharge coefficient, enhanced

spray-plume atomisation, minimal shot-to-shot variation, as well as exceptional durability considering cycling over several years (Karathanassis et al. 2020). It has been firmly established by the relevant research community that phasechange phenomena occurring in the internal flow-path of fuel injectors are strongly linked to the emerging spray morphology and dynamics (Payri et al. 2004; Mithun et al. 2018). The occurrence of cavitation, i.e. formation of vapour bubbles caused by flow depressurisation below the local liquid saturation pressure, is common in diesel fuel injectors, owing to the high-flow velocity and throttling during the needle opening/closing phases, as well as to the complex and geometrically constricted geometry of the device itself leading extensive flow recirculation to set in (Schmidt and Corradini 2001). To illustrate the topology evolution of in-nozzle two-phase flow processes, techniques to fabricate real-size (of the order of micrometres) injector tips from transparent materials and assemble them to modified injector metallic bodies have been developed in the last 30 years (Baik et al. 2003). Relevant visualisation investigations have been conducted at injection pressures ranging from a few hundred up to 2000 bar (Reid et al. 2014; Manin et al. 2020; Yasutomi et al. 2020). Parallel to those activities, several studies have focused on illustrating the in-nozzle, two-phase flow topology in enlarged nozzle-replicas at lower injection pressures, 
see selectively references (Andriotis et al. 2008; Gavaises et al. 2009), where flow similarity to the actual injector flow was ensured based on non-dimensional numbers. Distinct forms of cavitation have been identified by both types of experiments, broadly categorised as geometrically induced and vortical (or in some cases referred to as string) cavitation (Gopalan and Katz 2000; Arndt 2002). Of course, the nature of cavitation in high-speed flows is inherently transient and, thus, a multitude of distinct morphological features have been illustrated to encompass these two fundamental regimes, such as cavity shedding and elongated/ string cavitation (Laberteaux et al. 1998; Mishra and Peles 2005; Foeth et al. 2006).

To date, internal-nozzle optical flow visualisations predominantly rely on Mie-scattering from gas-liquid interfaces, facilitated by the use of transparent nozzle material with a refractive index similar to that of the fuel. Scattered light may be collected at an angle relative to the illumination source (e.g. side-scatter) or the extinction of light along the line-of-sight may be collected in a backlit imaging configuration (Aleiferis et al. 2010; Dumouchel et al. 2013; Hult et al. 2016). As has been discovered for imaging of the spray region, Mie-scatter illumination from the side presents many challenges, such as non-uniformities in lighting intensity exacerbated by optically thick regions (Pickett et al. 2015). Extinction imaging is therefore preferred, but this technique must utilise sufficiently diffused sources of backlit illumination to become insensitive to vapour-phase beam for sprays in highly evaporative high-pressure environments (Westlye et al. 2017). A variety of sources have been reported in the literature as suitable for backlit illumination ranging from halogen floodlights to LEDs, while incoherent light focused on the region of interest is usually achieved by a combination of engineered diffusers and Fresnel lenses.

Furthermore, X-ray imaging, especially utilising highflux synchrotron radiation, has been recently demonstrated as an attractive imaging technique, once again regarding both in-nozzle flow, even in steel injectors, and outlet nearfield (Kastengren et al. 2014; Moon 2016; Moon et al. 2016). The main advantage of X-ray imaging against conventional optical techniques lies on the weaker interaction of X-ray radiation with matter compared to visible light, thus allowing the visualisation of regions of high optical density (Karathanassis et al. 2018a). Focusing on the internal flow of fuel injectors, refractive index gradients in all the aforementioned techniques set in due to the presence of liquid/vapour interfaces, hence the underlying velocity field cannot be assessed. Nevertheless, an illustration of the in-nozzle level of turbulence and coherent secondary-flow patterns is crucial for the evaluation of the device performance, since LES have highlighted their strong independence with cavitation and, consequently spray evolution (Ghiji et al. 2017; Blume et al. 2019; Koukouvinis et al. 2020). It has to be noted that micro-PIV techniques which are, in principle, suitable for the flow-field illustration in microfluidics devices have scarcely been applied to optical injectors (Chaves et al. 2010; Kolokotronis et al. 2010; Hayashi et al. 2012; Mamaikin et al. 2020). Intrinsic technical difficulties associated with the achievement of a thin laser sheet compared to the injector dimensions have led to the adoption of diffused backlight illumination instead. Additional complexities arise due to the interaction of cavitation with seeding particles (Mamaikin et al. 2020). Hayashi et al. (2012) have demonstrated that a coherent recirculation pattern sets in in the sac region of a real-size optical diesel injector, while Mamaikin et al. (2020) revealed the flow separation occurring in the hole entrance of a transparent two-hole gasoline injector.

Schlieren imaging, a technique implemented in such a manner to be sensitive to refractive-index gradients, has been extensively utilised with reference to systems that exhibit steep density gradients, such as compressible gas flows, fluid mixing and vaporising liquid sprays. Consistently examined flow configurations constitute the cross-flow of jets, either gas or liquid, and a supersonic gas stream (Ben-Yakar and Hanson 2002; Beloki Perurena et al. 2009), as well as the supersonic expansion of gaseous jets (Phalnikar et al. 2008). Distinct shock-cell patterns arise in both flow scenarios that have been demonstrated to be faithfully resolved by the developed micro-schlieren set-ups. Phalnikar et al. (2008) aiming to visualise the shock-cell system of expanding gas micro-jets observed horizontal streaks distorting the schlieren structures. The elongated refractive-index gradients were assumed to correspond to a vortical motion introduced by imperfections on the nozzle surface. Microschlieren has been also successfully applied to quantify density gradients arising in diverse types of flow conditions and media, for instance, in the wake of a microsphere settling in a density-stratified liquid (Yick et al. 2006), during the mixing of a gaseous jet with stagnant ambient of different density (Tan et al. 2015), as well as during the mixing of liquids in micro-mixers (Sun and Hsiao 2013). With reference to fuel injection equipment, schlieren imaging is capable of elucidating the vaporisation processes taking place in the spray region, air entrainment as well as shockwave dynamics (Pastor et al. 2012; Vera-tudela et al. 2020). Such types of experiments have been conducted in different designs of constant-volume chambers replicating the high pressure and temperature conditions prevailing in the cylinders of internal combustion engines.

Turbulent structures arising in shear layers and wake flows can also be captured by schlieren imaging. Kouchi et al. (2015) implemented a schlieren system with a narrow depth of focus and managed to capture both shock and expansion-wave dynamics, as well as regions of increased turbulence in a dual-mode scramjet. Kirsch et al. (2019) discerned schlieren-like structures in a real-size, single-hole, 
transparent injector orifice despite employing diffuse-light illumination. Mauger et al. (2012) examined both a shadowgraph-like technique and schlieren imaging for the illustration of cavitation structures and shockwave dynamics in a quasi-2D mm-sized rectangular orifice. Refractive index gradients were observed in the shear layers forming between the attached cavities and the main-flow freestream at the channel inlet, as well as in the boundaries of the jet exiting the orifice; yet, intermittently, also along with the main flow.

The present study is, to the authors' knowledge, the first in the open literature demonstrating the capability of microschlieren imaging to illustrate the distinct vortical structures that develop in the internal flow path of a real-size diesel injector. Although this work employs a simplified, singleorifice injector geometry, the fundamental in- and nearnozzle flow phenomena elucidated, such as vortex roll-up mechanisms and spray dynamics, are highly relevant to the flow conditions prevailing in multi-hole injectors as well. The low-pressure regions forming at vortex cores induce refractive-index gradients that the developed micro-schlieren optical layout has the capability to capture even in the liquid in-nozzle flow. It has been confirmed that the geometricalconstriction of the fuel injector considered for the investigation, along with the transient needle motion, give rise to longitudinal vortices in the sac and orifice regions. Direct observation of these transient two-phase flow features of small length-scales requires high spatial and temporal resolutions. The study is also the first to use concurrent diffused backlit visualisation to determine when cavitation structures (gas-phase) appear and how either liquid vortical structures or cavitation lead to fluctuations in the cone angle of the expelled spray plume.

\section{Experimental setup}

\subsection{Injection system and pressure chamber}

The flow-visualisation experiments were performed in a sealed chamber, depicted in Fig. 1a. The cubical vessel has a characteristic dimension of $63.5 \mathrm{~mm}$, hence facilitating the use of short focal working distances for the optical system. Besides, line-of-sight optical access along two directions is offered through four fused-silica windows, $25 \mathrm{~mm}$ in diameter. The pressure within the vessel is controlled by imposing a nitrogen flow entering through four intake lines located at its top corners. A tube located at the bottom chamber wall serves as the exhaust for the fuel spray and nitrogen mixture. The ambient conditions examined lied in the range 1-20 bar, at a constant temperature of $20^{\circ} \mathrm{C}$. Commercial, multicomponent diesel fuel was used for the experiments pressurised to either 700 or 900 bar at the injector inlet by a high-pressure syringe pump. The injection and ambient pressure values were recorded by transducers located at the fuel line and the exhaust line in close proximity to the vessel, respectively.

The single-orifice transparent tip was mounted on a modified ECN Spray C/D injector metallic body. The metallic tip of the injector realising the injector holes was machined off below the needle seat and the optical injector assembly was accomplished by clamping the metallic body, optical tip and supporting pedestal into position. The pedestal has a hollow and corrugated layout to allow the visualisation of the spray region up to $1.5 \mathrm{~mm}$ downstream the injector outlet, as well as the propagation of the mixture into the exhaust tube. The transparent orifice was fabricated from cast acrylic due to its similar refractive index to diesel ( 1.45) and has a tapered-hole layout characterised by a $\mathrm{K}$ factor of $1.5\left(K=\left(d_{\mathrm{i}}[\mu \mathrm{m}]-d_{\mathrm{o}}[\mu \mathrm{m}]\right) / 10\right.$, where the subscripts correspond to the injector-hole inlet and outlet), while the hole entrance was also smoothened through hydro-erosion (Manin et al. 2020). The injector needle-lift profile was imposed by a signal with a duration of $1.2 \mathrm{~ms}$ provided by an electronic driver, which was kept identical for the two injection pressures examined. The injection duration, however, was dependant on the respective pressure, as made evident by Fig. 1b, owing solely to hydraulic phenomena designating the needle motion, which have also been reported by Manin et al. (2020). As can be seen, the overall event duration for $p_{\mathrm{inj}}=900 \mathrm{bar}$ is approximately equal to $2.85 \mathrm{~ms}$, about $0.2 \mathrm{~ms}$ longer than the respective for 700 bar. Besides, the needle-opening phase is shorter for the higher injection pressure examined, while also the injector operates at maximum lift for a larger time period, as made evident by the comparative lift values at around $t=1.0 \mathrm{~ms}$. It should be noted that the injection duration is considerably longer compared to the nominal of $1.5 \mathrm{~ms}$ employed by the ECN for diesel-spray investigations. The selected event duration was confirmed to be sufficient for the tracking of a multitude of schlieren structures arising in the nozzle, especially during the needle opening and closing phases, thus facilitating their statistical processing.

\subsection{Optical setup}

The layout of the optical set-up realised for the experiments is depicted in the schematic of Fig. 2. As can be seen, the DBI and schlieren systems are located in an orthogonal configuration by taking advantage of the dual-orientation optical access allowed by the chamber. Illumination is provided by two pulsating LEDs, namely one of blue colour for DBI and one of red for schlieren imaging, with radiation wavelengths of $455 \pm 22 \mathrm{~nm}$ and $620 \pm 19 \mathrm{~nm}$, respectively. The dye area for the red LED is $1.0 \mathrm{~mm}^{2}$ due to the necessity of a point source for the schlieren system, whereas a larger-dye LED $\left(24 \mathrm{~mm}^{2}\right)$ was employed for the DBI arrangement to allow 
Fig. 1 Experimental setup: a external (left) and section (right) views of the pressure chamber also depicting the geometrical layout of the transparent tip/pedestal assembly along with optical-microscopy image of the actual tip employed (dimensions in $\mathrm{mm}$ ). $\mathbf{b}$ Needle-lift profile for the two injection pressures examined $\left(p_{\text {back }}=1\right.$ bar $)$. The needle displacement at the final closing stages for $p_{\text {inj }}=900$ bar could not be detected due to cavitation obscuring the visualised region. The graph was produced by averaging the respective values of four injection events; shades encompassing the graph lines correspond to standard meanvalue error
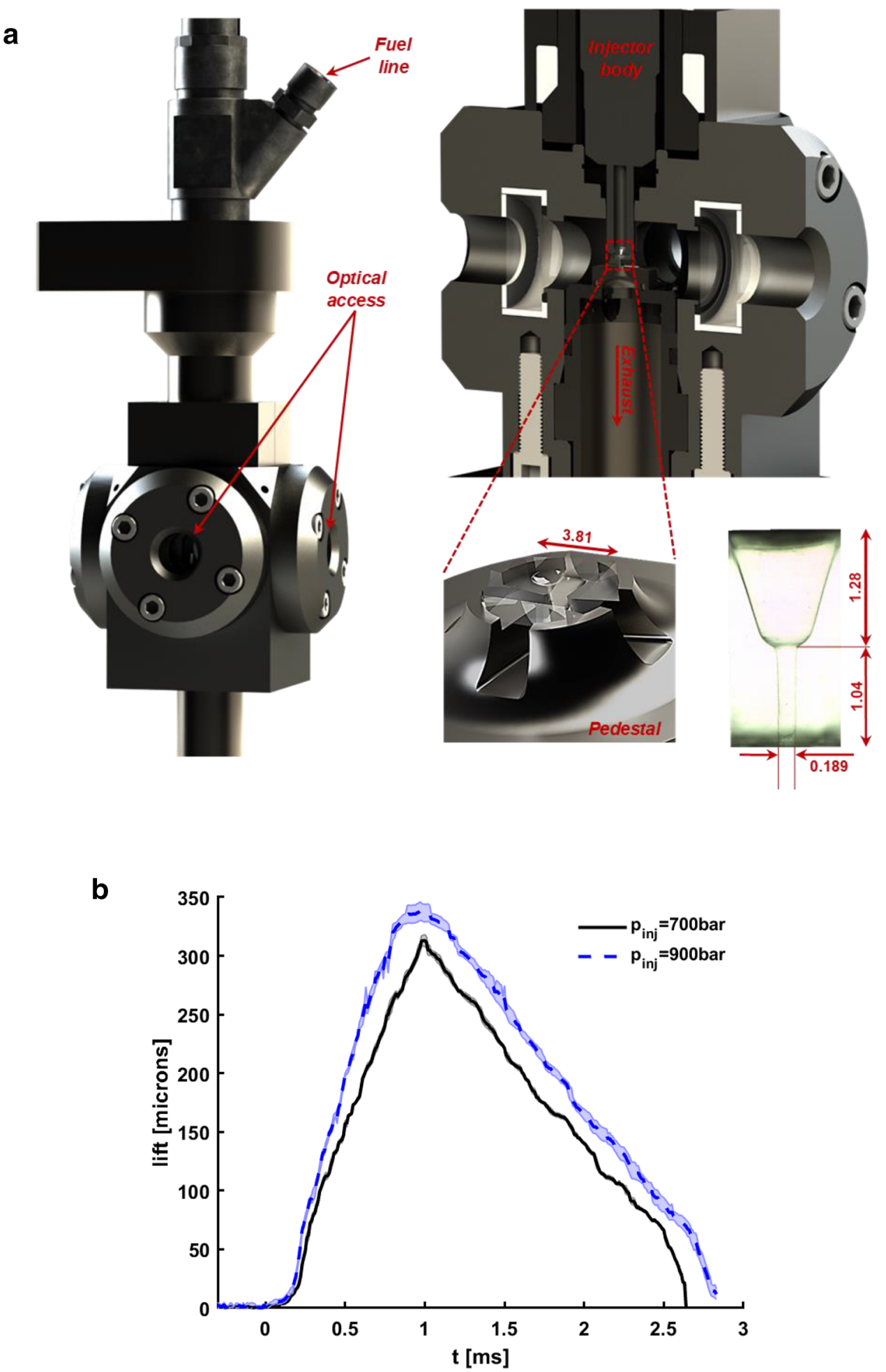

for ample illumination of the region of interest. A short pulse duration of $964 \mathrm{~ns}$, also designating the camera exposure time, was set for the red LED, so that the highly-transient schlieren structures can be tracked as faithfully as possible, while also allowing sufficient light to reach the camera sensor. A larger pulse width of $4 \mu$ s was imposed to the DBI source since preliminary tests showed that the arising innozzle cavitation predominantly exhibits static features, as clarified in the discussion relevant to Fig. 3a.

High-speed images were captured at 100,000 frames per second by two CMOS cameras (Photron SA-X2 and Phantom v2512) fitted with long-distance microscopes (Infinity 
Fig. 2 Schematic of the optical layout for the DBI and schlieren systems. Both LEDs/cameras are triggered by the injector driver, hence the same injection event is visualised concurrently by the two optical systems

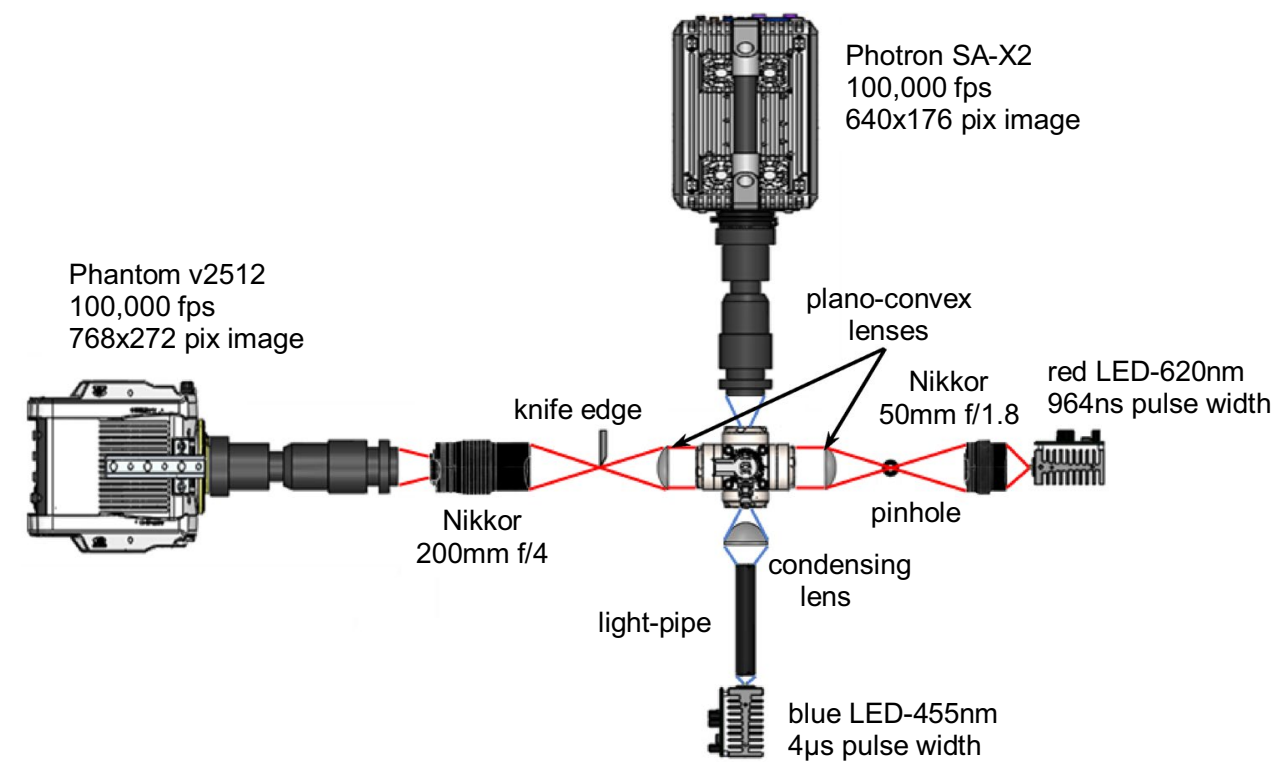

K2 with CF-2 objective and Mitutoyo $10 \times$ objective, respectively). A bandpass filter was fitted to the camera of the DBI system to prevent any wavelength cross-talk effects, whereas a filter was verified to be unnecessary in the schlieren system due to the small light-collection angle. The trigger-signal for the two cameras was provided by the injector driver, while, the LED pulse was fired at the mid-duration of the exposure time of each camera. The rest of the components comprising the optical system are also shown in Fig. 2. More specifically regarding the DBI system, the light beam passed through a light pipe acting as a diffuser and a condensing lens to minimise light spillage beyond the window of the vessel. With regards to the schlieren setup, a Nikkor $50 \mathrm{~mm}$ lens was used to focus the LED beam onto a pinhole, in essence acting as the point source of the system. A set of plano-convex lenses on each side of the region of interest was used to produce a parallel light beam and focus it on the knife-edge, respectively. The knife-edge was placed at a horizontal position, since longitudinal vortices were expected to arise within the orifice and, consequently, it is aimed to identify horizontal refractive-index gradients (the injector is placed at a vertical position, refer to Fig. 1a). A Nikkor $200 \mathrm{~mm}$ was placed in front of the long-distance microscope to bring the injector tip into focus. It should be clarified that a lens was preferred over a mirror (z-type) schlieren set-up, since it was established during preliminary experimentation with the optical set-up that the higher sensitivity of the latter rendered the sac region completely opaque merely due to the wall curvature. The plano-convex lenses with a certain level of spherical aberration (Settles 2001) eventually employed, along with the adjustment of the pin-hole active area modified the system sensitivity to enable the visualisation of structures in both the sac and nozzle regions.

\subsection{Obtained images and post-processing techniques}

The active visualisation window was discretised by $640 \times 176$ and $768 \times 272$ pixels, referring to the DBI and schlieren systems, achieving an image resolution of 3.90 and $3.24 \mu \mathrm{m} /$ pixel, respectively. A sequence of DBI images captured along the duration of an injection event is depicted in Fig. 3a, while the respective sequence of schlieren images is shown in Fig. 3b. A comparative assessment of the two panels makes clear that the refractive-index gradients setting in due to the presence of interfaces are much stronger compared to those owed to density gradients. Cavitation structures (Fig. 3a) are clearly discernible at the nozzle entrance, outlet and at localised regions along its length. Overall, it can be deemed that the nozzle is mildly cavitating and structures arise mainly due to manufacturing imprecision. For instance, a hole expansion during the removal of the cutting tool is common in conventionally-machined optical tips, hence the cavitation occurrence at the outlet region.

On the contrary, schlieren structures (Fig. 3b) are much more challenging to detect, partly due to the weak contrast of the refractive-index gradient itself but also due to the fact that light refractions caused by surface-roughness elements of the test-piece external side are being accentuated by the use of parallel light. However, elongated structures, as annotated in the time instances between 110 and $2410 \mu \mathrm{s}$ of Fig. 3b, appear in the sac region, emanating either from the gap between the needle and its seat (2210 and $2410 \mu \mathrm{s})$ or directly from the needle tip (310 and $510 \mu \mathrm{s})$ and are eventually entrained into the injector-hole. A multitude of transient structures with lifetimes close to the limit of visualisation capability also appear in the hole during the injector opening and closes phases, as illustrated in more clarity 
a
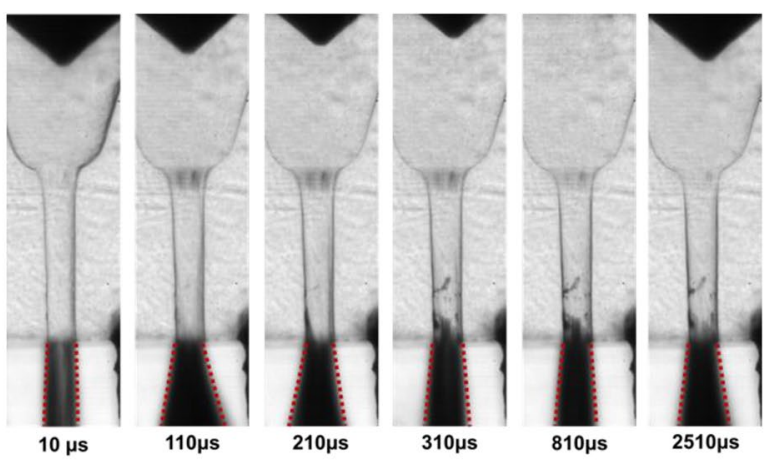

b
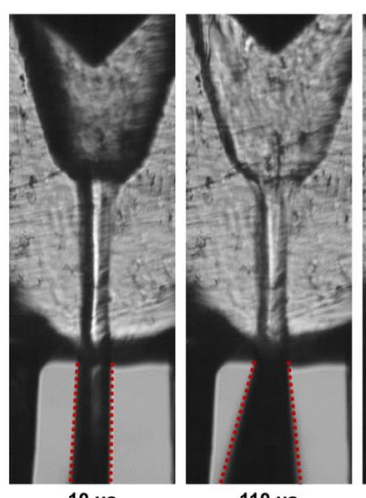

$110 \mu \mathrm{s}$

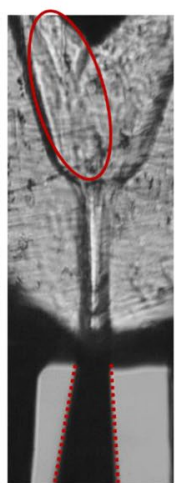

$210 \mu \mathrm{s}$
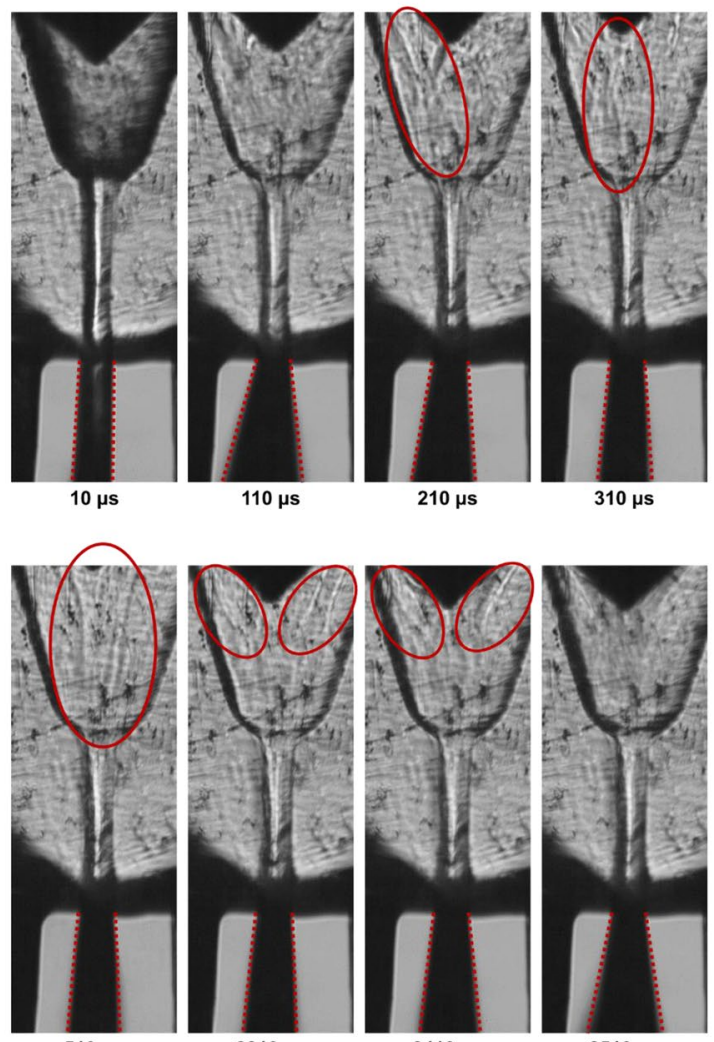

$510 \mu \mathrm{s}$

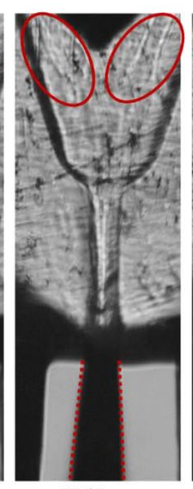

$2210 \mu \mathrm{s}$

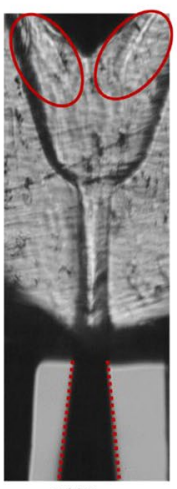

$2410 \mu \mathrm{s}$

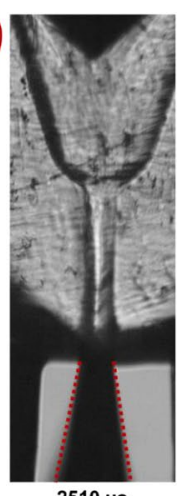

$2510 \mu \mathrm{s}$
Fig. 3 Sequence of high-speed images spanning throughout the injection event: a DBI and b schlieren imaging ( $900 / 5$ bar). Comparison of the time instances corresponding to $10 \mu \mathrm{s}$ and $110 \mu$ s makes clear that the internal flow path deforms as internal pressure is applied and the refraction-index gradient changes. The outline of the emerging spray has also been annotated to highlight the cone-angle variation during the event. Animations of the entire injection event are offered as supplementary material (movies SM1 and SM2)

by the animations offered as supplementary material (movie SM2). It is interesting to notice in Fig. 3, illustrating the near-nozzle spray region as well, that, although transient cavitation features cannot be detected, the spray-cone angle exhibits distinct fluctuations. The underlying flow processes will be elucidated in the results section.

A series of post-processing techniques were applied to the raw DBI and schlieren images to extract quantitative information concerning the extent of in-nozzle vaporous and vortical structures, as well as the spray-dynamics evolution. As already mentioned, the presence of an interface leads to strong refractive-index gradients and, hence, after the subtraction of a background from the raw images, a simple thresholding and binarisation technique was sufficient to identify the projected area of cavities.

However, the raw schlieren images required extensive treatment owing to the reasons discussed with relevance to Fig. 3b. At the first step, a dynamic background-subtraction process was implemented, in order to enhance contrast. Facilitated by the highly fluctuating behaviour of schlieren structures, it was verified that averaging a small number of consecutive images results to the extinction of any flowinduced gradients throughout the sac and nozzle regions. Image subtraction was therefore performed for each raw image by taking as a background the average of the previous 8 time instances so that the background geometry follows that of the treated image and artefacts due to flow-path mismatch caused by material deformation are minimised. As an additional means to de-noise the schlieren images, FFT filtering was applied to reduce periodic patterns arising, such as unphysical gradients aligned with distinct geometrical features, e.g. burs due to machining at the orifice entrance.

The phase congruency method, the theoretical formulation of which is described in detail in Kovesi (1995), was applied to detect the actual schlieren structures of interest in the enhanced-contrast images. In accordance with the formulation of the specific edge-detection technique, distinct image-features are identified by the phase and amplitude of the obtained signal in the frequency (Fourier) domain. Hence, the accuracy of phase congruency is not hindered by fluctuations in the image brightness, e.g. light-source flickering effects, or contrast, e.g. due to the small depth of focus of the employed lens. The method has been proven robust in detecting complex flow structures in X-ray imaging investigations, where the signal-to-noise ratio of the obtained radiographs is also low (Karathanassis et al. 2018a). After the application of phase congruency, the presence of refractiveindex gradients is highlighted, refer to the second panel of Fig. 4a. Small features are yet discernible, so the magnitude of horizontal (perpendicular to the main flow) brightness gradients is calculated to retain only longitudinal structures and subsequently the image is binarised using an adaptive thresholding procedure based on Otsu's method (Otsu 1979). The rather conservative thresholding ensures that strong, flow-induced refractive-index gradients are identified in the binarised image, yet along with the de-noising techniques applied, small-scale unrealistic features are totally absent, refer to the third panel of Fig. 4a. The final panel of the figure where the detected structures have been superimposed over the orifice geometry illustrates their onset in the sac region and entrainment into the injector hole. 
a

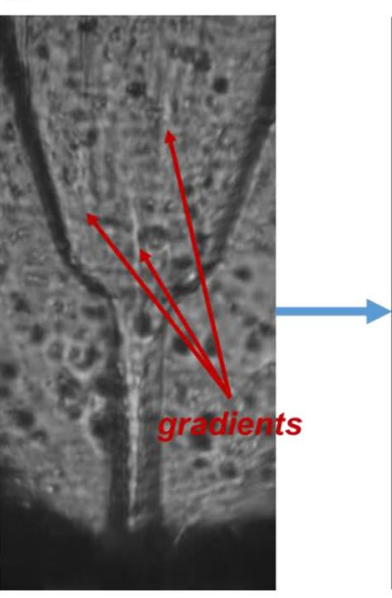

cropped raw schlieren image

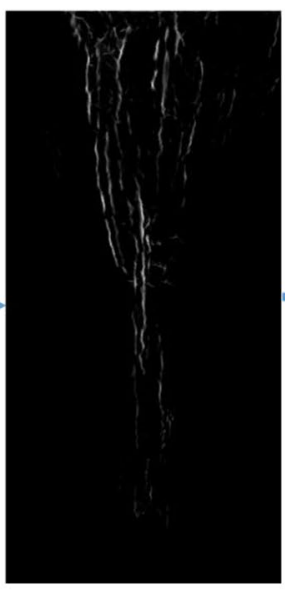

RI gradients detected by phase congruency

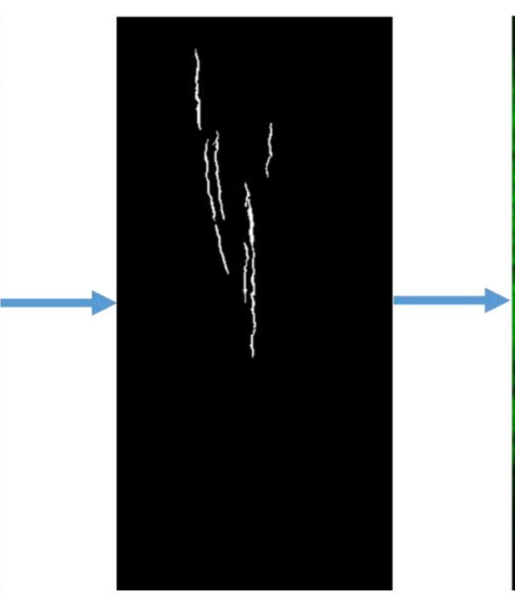

binarised image through adaptive thresholding

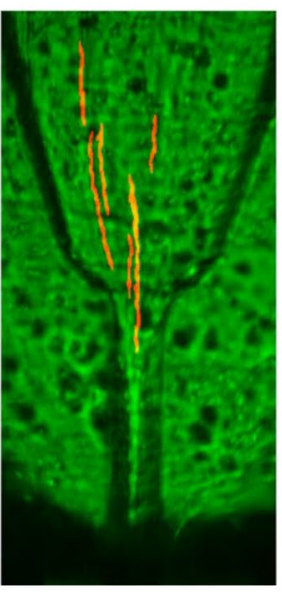

detected structures imprinted on raw image

b

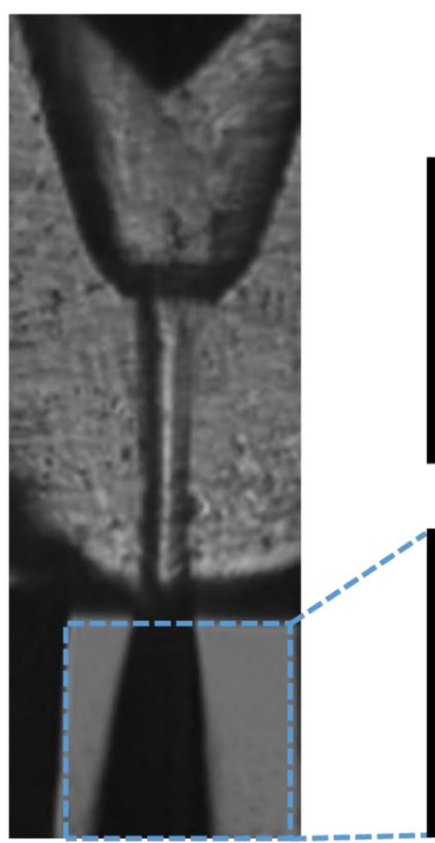

cropped image of the spray region approximation of identified regions with ellipses
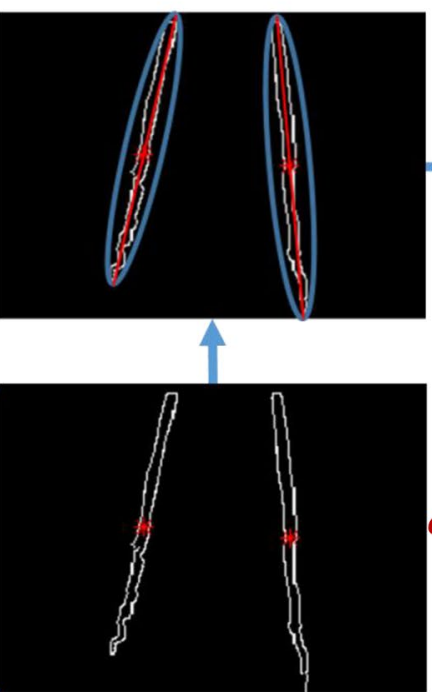

identification of
spray outline

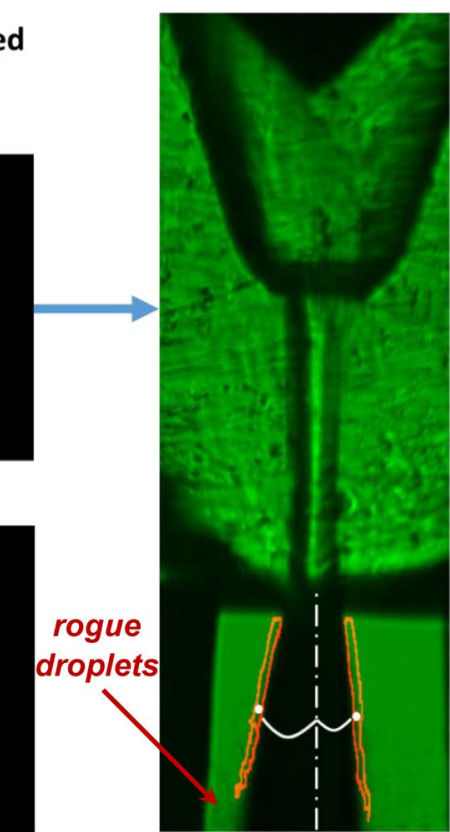

measurement of half-angles
Fig. 4 Basic steps of the post-processing techniques employed: a detection of schlieren structures and $\mathbf{b}$ measurement of the spray cone angle. The white dots denote the centroids of the region where brightness-gradient values are significant. Notice that even for the specific

Schlieren images offer a better contrast and higher resolution compared to the respective obtained through DBI and, thus, were also selected to extract information on the spray dynamics. The post-processing objective was to obtain the spray cone-angle variation with time. The basic steps flow conditions where the imperfections/spots on the part surface are quite discernible, they were successfully removed from the post-processed images

followed to detect the spray outline and measure the relevant cone angle are summarised in Fig. 4b. Initially, the brightness-gradient magnitude (along both directions) was calculated on a cropped region of the contrast-enhanced image encompassing the nozzle near-field. A careful look 
at the raw images, both in Figs. $3 \mathrm{~b}$ and $4 \mathrm{~b}$, suggests that, especially for diverging spray angles, the spray periphery contains detached droplets and locally exhibits a different contrast than the jet core. A faithful representation of spray divergence would necessitate these regions of reduced optical density to be captured to the maximum extent possible. Calculation of the pixel-brightness gradient magnitude highlights the spray periphery and a binarisation and edge-detection procedure (using a conventional algorithm, e.g. Canny's) leads to the formation of two characteristic closed-outline regions, as can be seen in the mid-panel of Fig. 4b. The identified regions of arbitrary shape were fitted with ellipses, the major axes of which were used to define the cone half-angles against the orifice symmetry axis. The advantage of this method over a 'point measurement' at a specific location, is that the perturbed outline of the spray is better reflected in the cone-angle values measured. It must also be noted that any 'rogue' droplet clouds evident in the

Table 1 Matrix of examined test cases The Reynolds number was calculated using the orifice outlet diameter $d_{0}$, as a characteristic length scale. Indicative properties for the diesel fuel $\left(v_{\mathrm{f}}\right.$ and $\left.p_{\text {sat }}\right)$ used for the definition of non-dimensional numbers were obtained by Kolev (2007)

\begin{tabular}{ll}
\hline Fuel & Multi-component diesel \\
\hline Ambient gas & $\mathrm{N}_{2}$ \\
Injection pressure $\left(\cdot 10^{5} \mathrm{~Pa}\right)$ & $700-900$ \\
Ambient temperature $(\mathrm{K})$ & 293 \\
Ambient pressure $\left(\cdot 10^{5} \mathrm{~Pa}\right)$ & $1-5-20$ \\
Ambient density $\left(\mathrm{kg} / \mathrm{m}^{3}\right)$ & $1.15-5.76-23.09$ \\
$\operatorname{Re}\left(=\frac{u \cdot d_{0}}{v_{\mathrm{f}}}\right)$ & $15,200(700 / 20$ bar $)-17,500(900 / 1 \mathrm{bar})$ \\
$\mathrm{CN}\left(=\frac{p_{\text {inj }}-p_{\text {back }}}{p_{\text {back }}-p_{\text {sat }}}\right)$ & $34(700 / 20$ bar $)-1,138(900 / 1$ bar $)$ \\
\hline
\end{tabular}

active window, refer to the annotation on the raw image of Fig. 4b, have been filtered out and do not obscure the detected spray outline.

\section{Results}

The results presented in this section have been produced by the matrix of experimental cases summarised in Table 1 and have been derived by at least five injection events recorded for each one of the examined test cases. A maximum injection pressure of 900 bar was selected, which ensures that the optical tip can withstand multiple injections without failing, whereas the chamber pressure was adjusted between atmospheric pressure and 20 bar, the latter corresponding to a medium-density matching that of the standard spray A condition (ECN 2020). The ambient was maintained at room temperature for all examined test cases.

\subsection{In-nozzle cavitation and vorticity}

To highlight the overall extent of in-nozzle cavitation and its dynamic evolution, the projected vapour area has been averaged during the entire injection event and the relevant values along with their standard deviation are presented in Fig. 5. It has to be emphasised that, since optical imaging is employed, insight on the volumetric extent of the structures, i.e. whether they occupy the entire orifice or just its periphery, cannot be given with certainty but the main aim is to verify if considerable transient features prevail during the event. The contour plots of Fig. 5 corresponding to the two examined injection pressures demonstrate the opposite, namely that the orifice, due to its tapered hole layout, cavitates very mildly with the most extensive structure located at the outlet region. The specific cavity remains static as
Fig. 5 Extent and dynamics of cavitation determined through DBI for different injection pressures: a mean probability and b standard deviation of vapour presence. The black line indicates the outline of the injector hole. Cavities do not appear in the sac region throughout the event; hence, zero values dominate the entire upper part of the plots

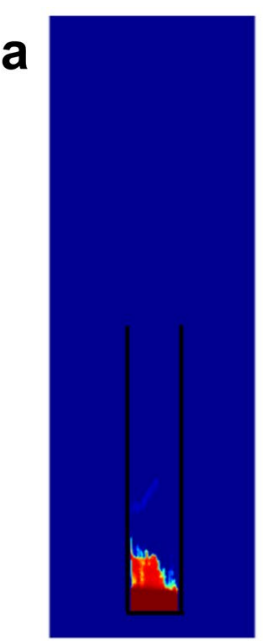

700/1 bar

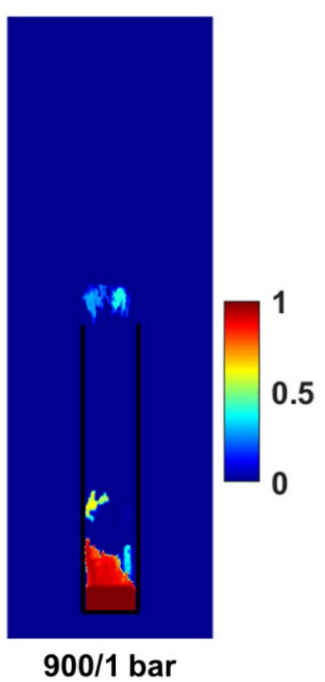

900/1 bar

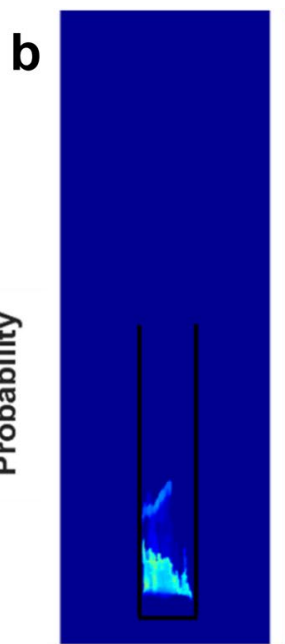

700/1 bar

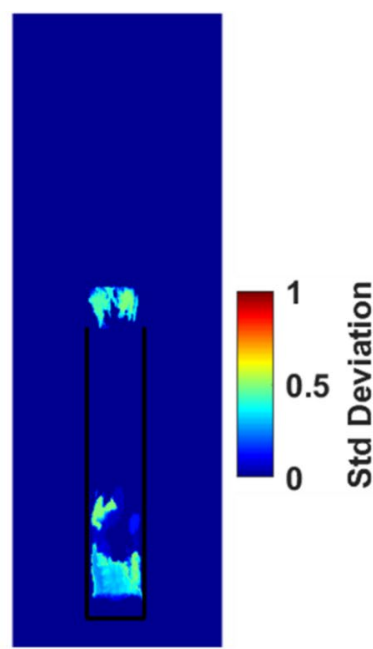

900/1 bar 
indicated by the high probability values and expands slightly as $p_{\text {inj }}$ increases. Additional minor cavities can be detected at the orifice entrance and slightly downstream its mid-length, which are more pronounced for $p_{\text {inj }}=900$ bar. These localised cavities do exhibit a transient nature, in the sense that they are not present throughout the injection duration, yet the relevant standard-deviation image (Fig. 5b) makes clear that extensive transient vaporous structures are absent in the major part of the orifice. Mild structure shedding can be detected between the localised cavity (possibly stemming from a manufacturing imperfection) and the outlet cavity, as indicated by non-zero standard deviation values between the two regions. Since no appreciable cavitation dynamics can be detected, only the contour plots for atmospheric backpressure have been presented for brevity, as they constitute the most extreme conditions, in terms of $\mathrm{CN}$, for each $p_{\text {inj. }}$.

The distribution of the overall projected vapour area in the injector hole during the injection event is depicted in Fig. 6. As mentioned in Sect. 2.1, the injection duration is dependent on the upstream pressure and therefore, to offer a more straightforward comparison of flow-processes evolution for different conditions, time is presented in a nondimensional form. As expected both injection and back-pressure affect the extent of in-nozzle vapour formation, since, in essence, designate the $\mathrm{CN}$ value. Injection into atmospheric conditions (Fig. 6a), is, in general associated with a higher extent of in-nozzle cavitation compared to the respective in a high-pressure environment (Fig. 6b). What is important to notice though, is that the projected vapour area remains relatively constant for the, so called, steady part of the injection, i.e. $0.2<t^{*}<0.8$ regardless of the prevailing conditions. The absence of strong transient cavitation structures is once again demonstrated.

Having a rationale similar to that reported regarding Fig. 5, the contour plots presented in Fig. 7 represent the averaged probability of schlieren-structure presence a

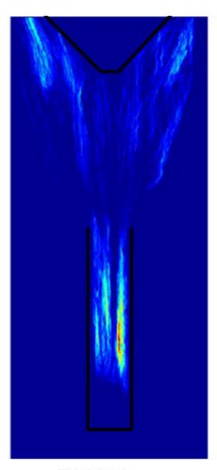

700/1 bar

b

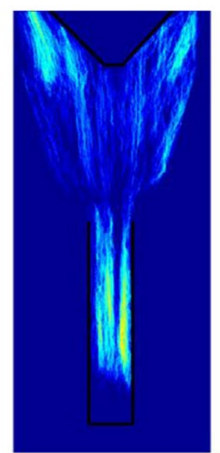

700/1 bar

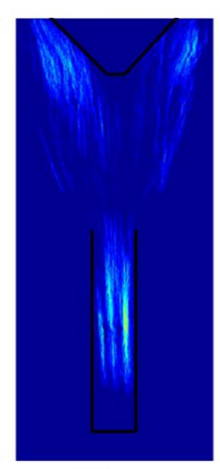

$700 / 5$ bar

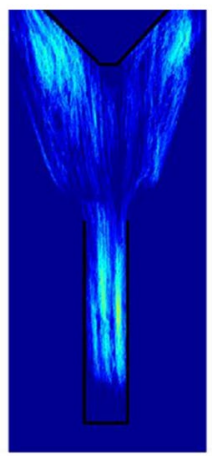

$700 / 5$ bar

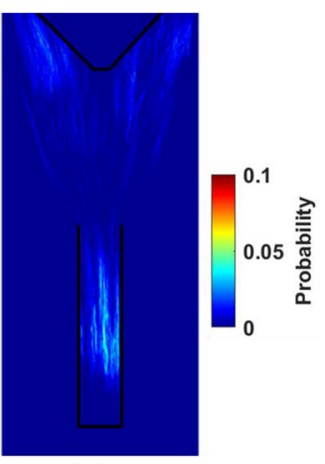

$700 / 20$ bar

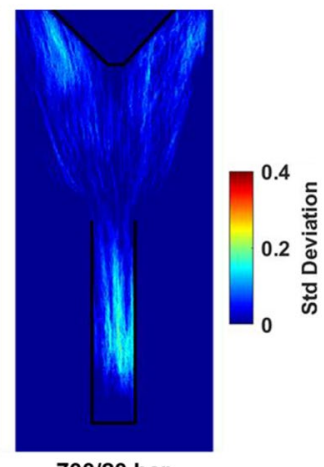

$700 / 20$ bar
Fig. 7 Extent and dynamics of schlieren structures for $p_{\text {inj }}=700$ bar: Contour plots of the $\mathbf{a}$ mean probability and $\mathbf{b}$ standard deviation of structure presence. The black line denotes the indicative extent of the injector hole and of the needle at its lowest position

throughout the duration of the injection event (Fig. 7a) and relevant standard deviation (Fig. $7 \mathrm{~b}$ ) for $p_{\text {inj }}=700$ bar and $p_{\text {back }}=1-20$ bar. A noteworthy initial observation is that the probability values are quite low reaching up to $10 \%$ for injection into the atmosphere, indicative of the highly-transient nature of the underlying flow processes. The highest probability and standard deviation values are encountered
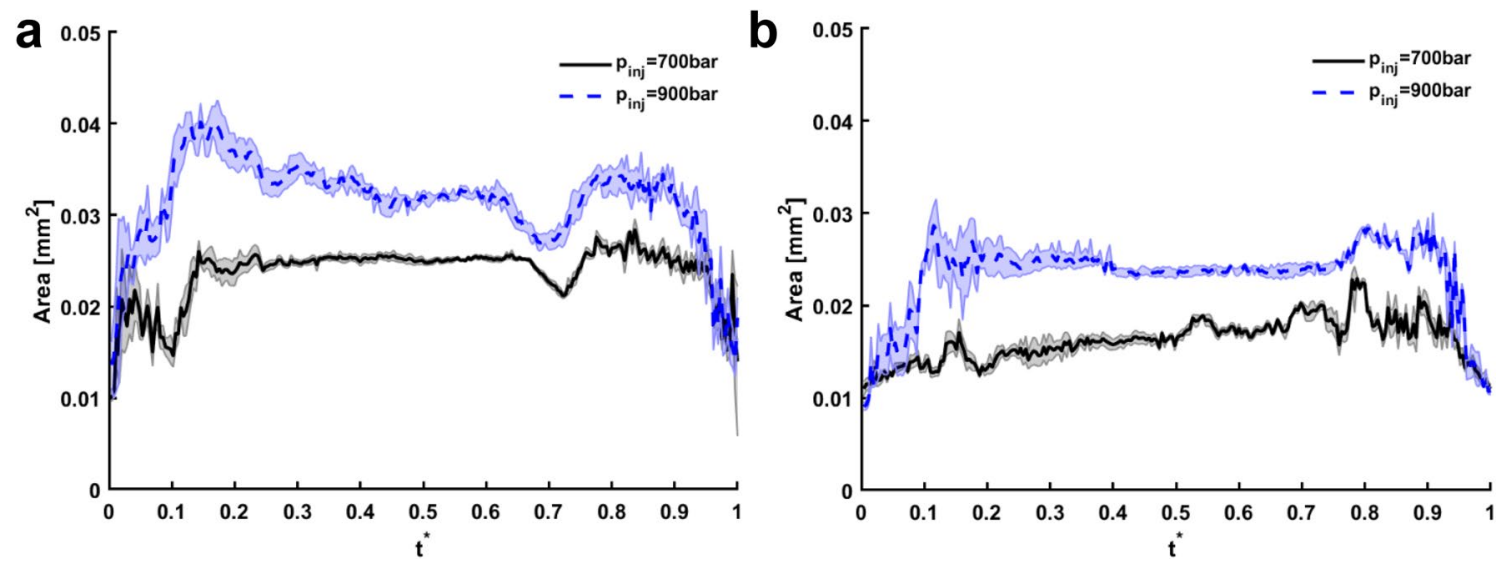

Fig. 6 Effect of injection and back pressures on the projected vapour area: $\mathbf{a} p_{\text {back }}=1$ bar and $\mathbf{b} p_{\text {back }}=20$ bar. The non-dimensional time has been defined as $t^{*}=t / t_{\mathrm{tot}}$, where the total injection duration $t_{\mathrm{tot}}$ can be obtained from Fig. $1 \mathrm{c}$ 
in the injector hole and, in fact, in three localised regions occupying the major part of the hole cross-section. Increase of the back-pressure, affecting the Reynolds number that characterises the internal flow but also slightly the needlemovement dynamics (Manin et al. 2020), leads to a moderate decrease in the extent of schlieren structures in the sac and nozzle regions. This behaviour suggests that the onset of density gradients is primarily owed to flow-dynamics processes rather than temperature gradients. A brief comment can be made for the lack of identified structures in the nozzle outlet region. As also indicated in Fig. 3, the specific region is completely opaque for the schlieren system owing to both in-nozzle cavitation and local material deformation.

In a similar manner, contour plots of the average presence probability and standard deviation of schlieren structures for the highest injection-pressure examined, $p_{\text {inj }}=900 \mathrm{bar}$, are shown in Fig. 8. Clearly discernible differences can be detected between Figs. 7 and 8 corresponding to the two injection pressures, with the most distinctive being the multitude of structures in the injector hole (region A). A single dominant structure prevails in the hole for $p_{\text {inj }}=900$, a pattern, which is, in fact, consistent regardless of the

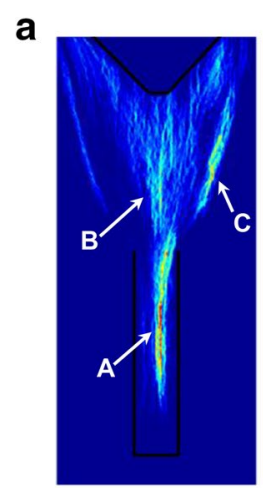

900/1 bar

b

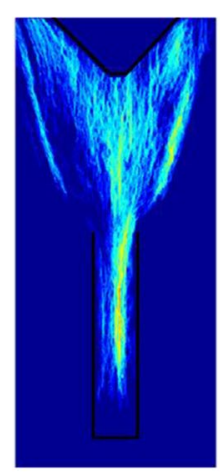

900/1 bar
$900 / 5$ bar

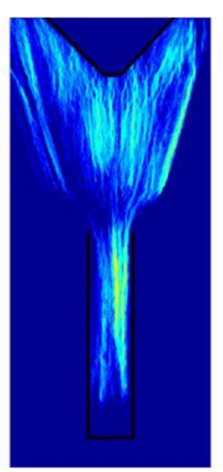

$900 / 5$ bar

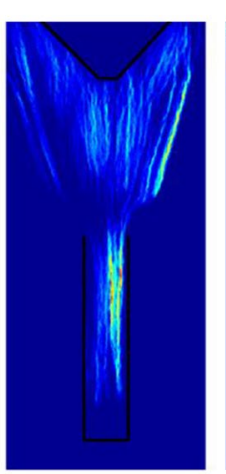

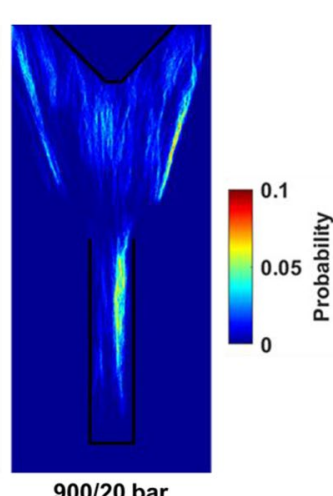

$900 / 20$ bar

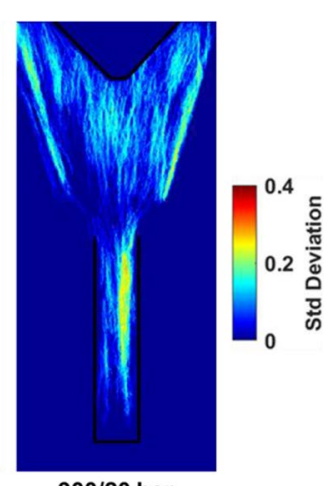

900/20 bar
Fig. 8 Contour plots of $\mathbf{a}$ mean probability and $\mathbf{b}$ standard deviation of schlieren (vortical)-structure presence for $p_{\text {inj }}=900$ bar. Characteristic regions $(\mathbf{A}-\mathbf{C})$ correlated with distinct underlying processes have been annotated on the plots ambient pressure. Besides, the region of maximum presence-probability values is more extensive in Fig. 8a compared to Fig. 7a. Especially the standard deviation values (Fig. 8b) demonstrate that increase in the injection pressure enhances significantly the manifestation of incoherent structures (since the probability values remain low) in the entire sac region. Focusing on the case corresponding to the pressure ratio of $900 / 1 \mathrm{bar}$, which exhibits the largest extent of schlieren structures, one can discern a high-probability region (B in Fig. 8a) bridging the needle tip with the coherent structure in the injector hole. The same region is also highlighted by the locally high standard-deviation values. Distinct characteristics of the schlieren structures manifestation, such as high sensitivity to the injection/back pressures, transient nature and emergence in the sac/nozzle core provide ample justification to the argument that the underlying density gradients form due to the onset of longitudinal vortices. The second Helmholtz theorem stating that a vortex filament must extend to the fluid boundary or form a closed path is also in support of this observation (Batchelor 2000). It is clearly illustrated in the schlieren-images animation (movie SM2) and especially in the time instances between 900 and $1100 \mu$ s that an elongated structure, in essence a longitudinal vortex, emanates from the region of the needle tip and propagates into the injector hole. Although the vast majority of schlieren structures exhibit a transient nature, a remark must be made for the refractive-index gradients arising adjacent to the sac wall. A streak of high-probability values is also evident (region C) in all panels of Fig. 8a. Indeed, it is highly plausible that temperature effects, i.e. viscous heating in the boundary layer (Koukouvinis et al. 2020), also contribute to the refractive-index gradient arising in region C. In general, referring to compressible liquids, the influence of temperature on density is considerably stronger to that of pressure. A temperature difference of few degrees, plausible to arise in injector flows due to viscous heating, causes similar density variations as a pressure difference of tens of bar (Mauger et al. 2012). Another potential contribution to the refractive-index gradient in region $\mathrm{C}$ constitute longitudinal vortices locally forming by Goertler-type instabilities due to the concavity of the wall (Saric 1994).

Figure 9 contains information on the projected-area distribution of the vortical/schlieren structures with time throughout the injection event. It must be noted that the graph values correspond to the structures detected both in the sac and nozzle regions. A much more fluctuating distribution can be discerned compared to the respective regarding the vapour area presented in Fig. 6. A multitude of longitudinal vortices do appear during the injection event, yet they are not strong enough to cause the fluid to cavitate. In-nozzle vorticity obtains its maximum extent for the highest $p_{\text {inj }} / p_{\text {back }}$ ratio of $900 / 1$ bar, while increase of $p_{\text {back }}$ has a mild hindering effect. Besides, the averaged 

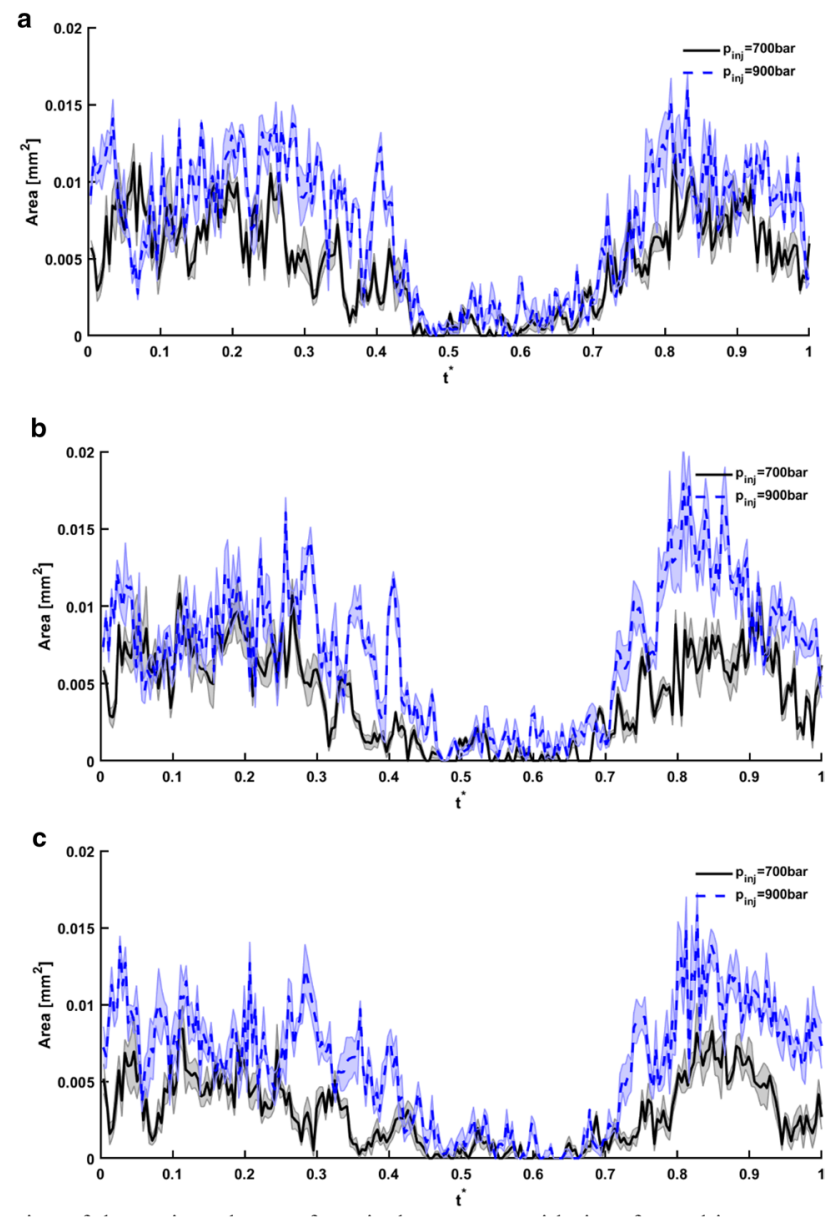

Fig. 9 Distribution of the projected area of vortical structures with time for ambient pressure of: a 1 bar, b 5 bar and c 20 bar. The projected area in each time instance corresponds to the area-sum of structures identified in the binarised images (refer to Fig. 4a)

in-nozzle extent of vortical structures appears reduced by $34-52 \%$, on average in the range of $p_{\text {back }}=1-20$ bar, for $p_{\text {inj }}=700$ bar compared to 900 bar. However, it must be also noted that the projected vortex-area values are associated with relatively high statistical error, as indicated by the shaded region on Fig. 9. The extent of vortices is, once again, essentially correlated to the prevailing flow conditions; an increase of the injection pressure leads to higher in-nozzle velocities but also to a more-transient opening phase influencing the flow blockage ratio at the region of constriction, refer to Fig. 1b. Both influencing factors are, indeed, anticipated to enhance the manifestation of longitudinal vortices, thus further corroborating the statement that schlieren structures set in due to coherent recirculating motion. As expected, the extent of structures is higher during the opening and closing phases of the cycle, vortices nonetheless are present even for operation under high needle lift, indicatively for $0.4<t^{*}<0.6$.
Considering that the flow lies well in the turbulent regime, based on the nominal Reynolds-number values shown in Table 1, but also the inherent features of geometrically constricted flows, i.e. three-dimensional separation and onset of longitudinal vortices (Wilhelm et al. 2003), it becomes evident that vortical structures at different length scales interact within the injector. It is therefore of interest to examine the level of vorticity separately in the sac and nozzle regions since propagation of longitudinal vortices in locations close to the injector outlet is plausible to affect the spray dynamics. Figure 10 presents the ratio of vortical-structure presence probability in the injector hole over the respective values in the sac. Each probability value was derived by spatially averaging the probability distribution in each characteristic region, as shown in the inset of Fig. 10a. It is evident that increase of the injection pressure leads to a significant decrease in the presenceprobability ratio. In other words, although much more vortical structures appear in the sac region for $p_{\text {inj }}=900 \mathrm{bar}$ compared to 700 bar (refer also to the contour plots of Figs. 7, 8 and the relevant discussion), these structures do not eventually propagate into the nozzle. Increase of the injection pressure brings about an increase to both the Reynolds number and the overall in-nozzle level of turbulence, i.e. vortices at scales impossible to be visualised with the current setup. Hence, the interaction of vortices at different scales obtains a competing nature, a fact that has been established in injector flows (Karathanassis et al. $2018 \mathrm{~b}$ ). Strong and stable longitudinal vortices manage to prevail in the injector hole, while transient structures of lesser magnitude are eradicated due to viscous decay. As an overall outcome, for the highest injection pressure examined, large-scale vortical structures that are present in the injector hole are expected to exhibit a more static spatial topology, whereas for the lowest injection pressure more fluctuating structures manage to propagate or set in in the region, which is also confirmed by the contour plots of Figs. 7 and 8.

The pseudo-colour images, corresponding to early time instances of the injection event, depicted in Fig. 10b, c highlight the presence of the very few strings cavities that could be detected. Since they appear close to the outlet, it cannot be deduced with certainty whether the underlying vortices cause nucleation due to their increased magnitude or due to the fact that they retain their coherence for longer potentially entraining gas from the ambient, in both cases owing to the reduced interaction with turbulence. In any case, the strings act as markers for the underlying vortical motion and further support the conclusion that more coherent vortices are plausible to manifest themselves across the nozzle cross-section for the low (Fig. 10b), rather than high injection pressure (Fig. 10c). 
Fig. 10 a Presence probability ratio $(=\mathrm{P} 1 / \mathrm{P} 2)$ of vortical structures in the hole (probability P1) over the sac region (probability P2). Pseudo-colour, to enhance the contrast of cavities, DBI time instances during the early stages of the injection event: $\mathbf{b}$ 700/1 bar, c 900/1 bar. The $t^{*}$ values exhibit minor differences due to the dependence of injection duration on pressure

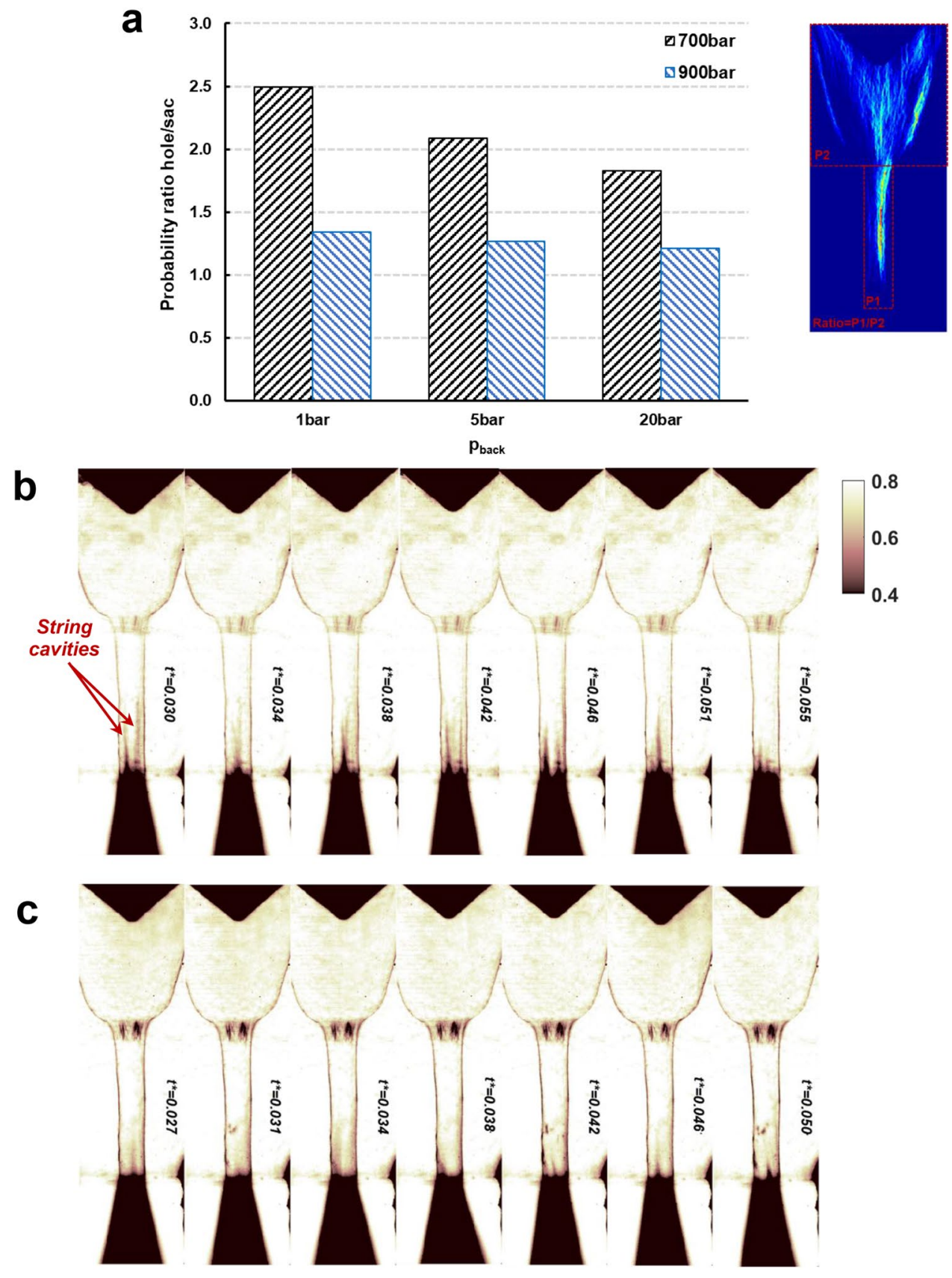

\subsection{Spray dynamics and correlation to in-nozzle flow}

As already mentioned, in-nozzle flow conditions can have a crucial impact on the topology and evolution of the emerging spray. Hence, the elucidation of linking flow processes is important for the performance evaluation of the injector. Figure 11 presents the spray cone-angle variation with time for the various injection- and back-pressures examined. In general, it can be discerned that the cone angle exhibits a similar qualitative distribution for the low and high injection pressures, with increasing back-pressure leading to a higher average cone angle. This behaviour should be attributed to the dominant effect of drag for a high-pressure ambient, especially for $p_{\text {back }}=20$ bar (Fig. 11c). For lower back-pressures (Fig. 11a, b), the cone-angle values are higher and their distribution is somewhat more fluctuating for $p_{\text {inj }}=900$ bar, as also demonstrated by the higher values of statistical uncertainty. The underlying cause should be sought to increased in-nozzle perturbations (refer also to the discussion regarding Fig. 10) being reflected on the spray dynamics.

A spray flapping sequence can also be discerned for $t^{*}=0.2-0.8$, where the cone angle exhibits smooth 
a

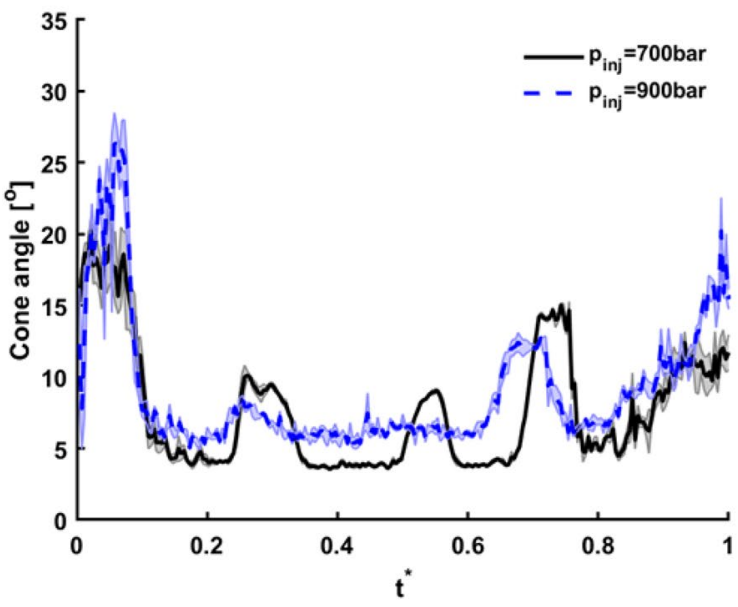

b

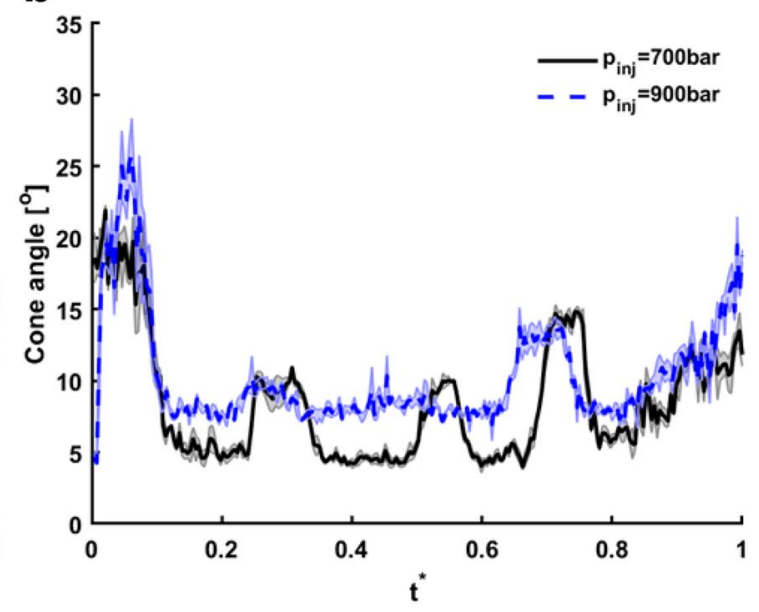

C

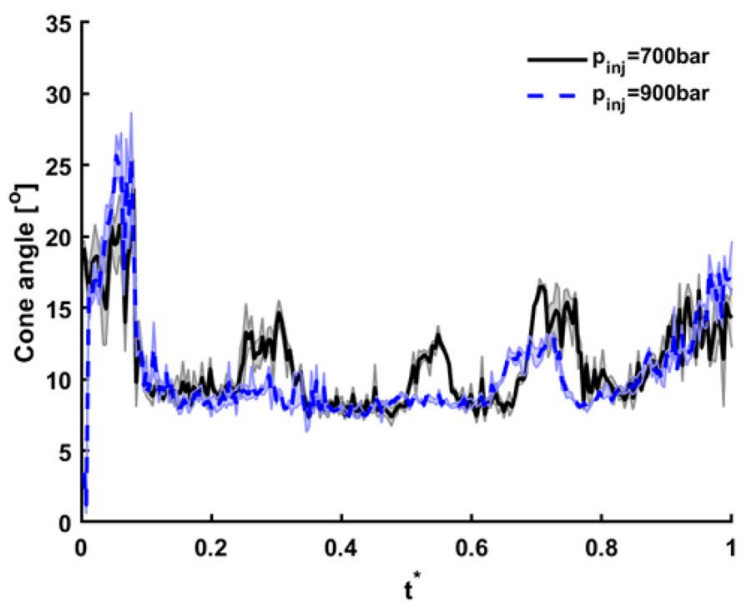

Fig. 11 Spray cone-angle distribution with time: ambient pressure of a 1 bar, $\mathbf{b} 5$ bar and $\mathbf{c} 20$ bar

alternations of high and low values. The local maxima are not fully matched in the two distributions, yet this is expected due to the needle-lift profile being dependant on the injection pressure. A distinct discrepancy is evident at the event mid-duration, between $t^{*}=0.5-0.58$, which is, in fact, consistent regardless of the back pressure; the cone angle distribution systematically exhibits higher values for the lower injection pressure of 700 bar. Since the $t^{*}$ values correspond to maximum lift and transient cavities are totally absent during that period, it is postulated that the in-nozzle vortical motion has a clear effect on spray flapping.
Figure 12 and the accompanying animations provided as supplementary material (movies SM3-SM6), focus on the time period between $t^{*}=0.50$ and 0.58 , where the distinct spray flapping arises only for the 700 bar injection. Successive time instances of phase congruency intensity are depicted, overlapping raw images showing the nozzle and spray regions. The phase congruency images corresponding to $p_{\text {inj }}=700$ bar (Fig. 12a, c, e) reveal that a strong refractive-index gradient prevails in the region of the right boundary layer, referring to the orifice cross-section projection. In fact, it has been verified that this behaviour is consistent 
regardless of the ambient pressure, as demonstrated by the relevant panels referring to $p_{\mathrm{amb}}=1$ bar (Fig. 12a), 5 bar (Fig. 12c) and 20 bar (Fig. 12e). It is also interesting to notice that the spray cone diverges asymmetrically with the expanding side coinciding with the radial location of the schlieren structure, as made evident especially in the supplementary movies SM3 and SM5. On the contrary, only a few scarce structures can be discerned on Fig. 12b, d, f corresponding to $p_{\text {inj }}=900 \mathrm{bar}$ and therefore the spray cone angle remains symmetrical throughout the examined time range. Nevertheless, movies SM4 and SM6 accompanying Fig. $12 \mathrm{~b}, \mathrm{f}$ elucidate that the minor structures that do arise within the nozzle actually cause rapid fluctuations in the cone angle. This oscillating trend is also reflected on the cone-angle distributions shown in Fig. 11 and also illustrated by the magnified view for $900 / 1$ bar, presented as an inset to Fig. 12a.

The flow mechanism proposed to link in-nozzle vorticity to spray dynamics is illustrated by the schematic of Fig. 13 . In essence, the presence of the longitudinal vortex, which gives rise to the refractive-index gradient, causes the formation of a shear layer as free-stream liquid interacts with a recirculating flow of reduced axial velocity. In turn, parallel flow is asymmetrically entrained past the vortex, which practically acts as an 'obstacle' located in the boundary-layer region, thus leading to a subsequent asymmetrical jet expulsion downstream the injector outlet.

\section{Conclusions}

This work provided insight on the correlation of the dynamics of the spray expelled by a single-orifice diesel injector with the cavitation and coherent recirculation pattern arising in its internal flow path. A DBI and a micro-schlieren system were employed to concurrently visualise the evolution of in-nozzle vapour and schlieren structures, as well as the fluctuations of the spray cone. The developed microschlieren system was demonstrated to be capable of capturing the highly-transient schlieren structures arising during the operating cycle of the injector. These structures were proven to set in due to the density gradients that form at the cores of longitudinal vortices rolling-up in the injector sac and nozzle or being entrained from the one region to the other. The nature of the refractive-index gradients was proven by their high sensitivity to the injection and ambient pressures and the fact that they occasionally emanated from the needle tip. It was found that the probability of a longitudinal vortex emerging or propagating in the injector hole decreased with increasing injection pressure. The trend was attributed to the interaction of large-scale coherent vortical motion with turbulence, i.e. eddies at length-scales that cannot be captured by the imaging system. As the in-nozzle level of turbulence increases, only strong, localised vortices survive in the injector hole.

Although, DBI data demonstrated that the orifice is only mildly cavitating due to its tapered layout and any transient cavitation structures are negligibly minor, still the spray topology exhibits significant fluctuations during the injection event. In fact, it was shown that the spray plume exhibited a characteristic widening at high needle lifts for the lowest of the two injection pressures examined (700 bar). This characteristic spray behaviour, which was absent for the higher injection pressure, is explicitly correlated to the presence of coherent recirculation pattern in the injector hole. In fact, the spray plume asymmetric expansion is linked to the radial location of the in-nozzle longitudinal vortex. From an optical-diagnostics point of view, the study demonstrates the robustness of the dual-view imaging technique on highlighting flow characteristics, which cannot be captured by 

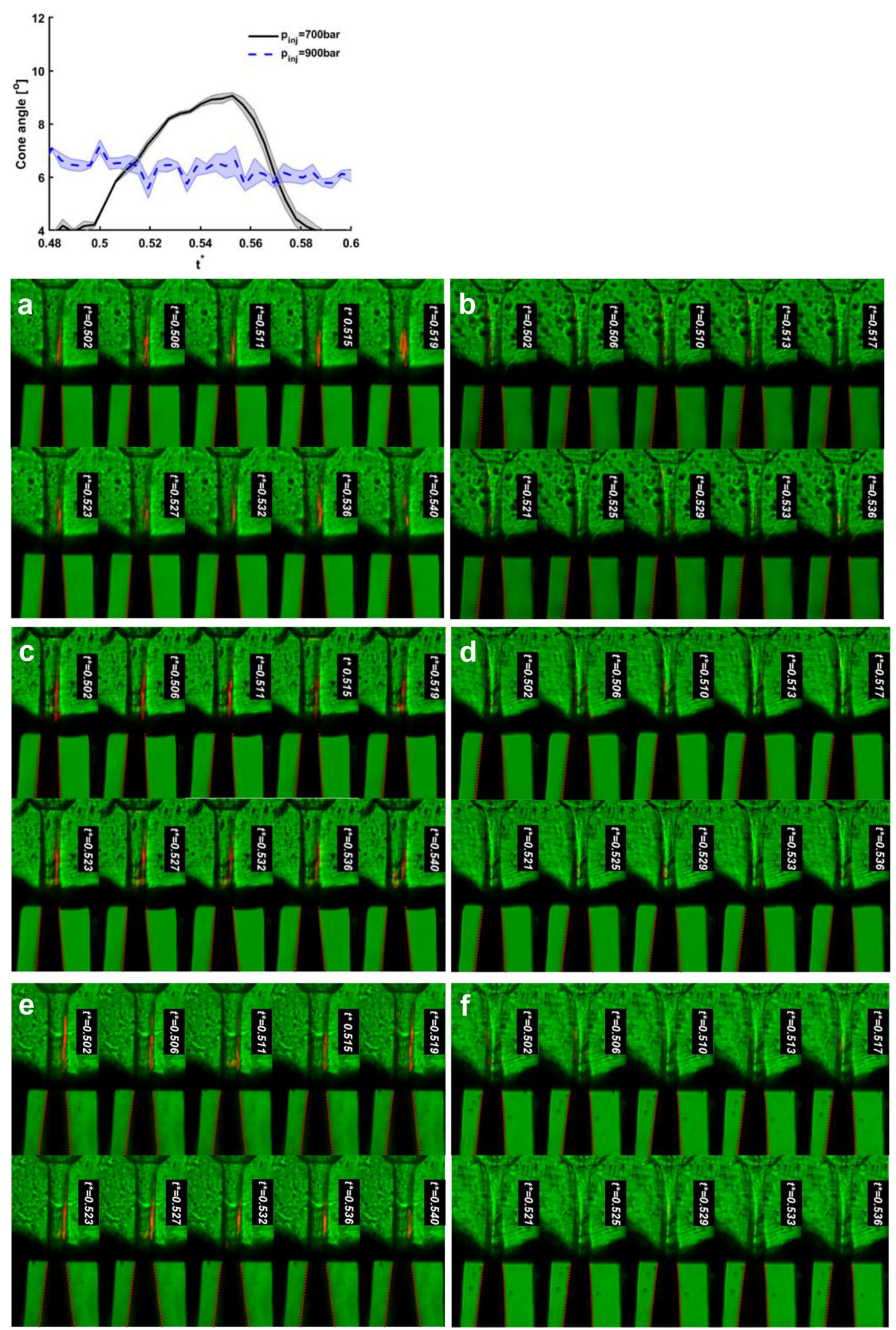
4Fig. 12 Correlation of in-nozzle flow with spray flapping at the midduration of the injection event: phase-congruency overlapping respective raw images also depicting the spray cone angle: a 700/1 bar, b 900/1 bar, c 700/5 bar, d 900/5 bar, e 700/20 bar and f 900/20 bar. Vortical (schlieren) structures are highlighted in red colour. The inset of a depicts the cone angle distribution for the time period in mention. Animations for the presented cases $\mathbf{a}, \mathbf{b}$ and $\mathbf{e}, \mathbf{f}$ are provided as supplementary movies SM3, SM4 and SM5, SM6, respectively

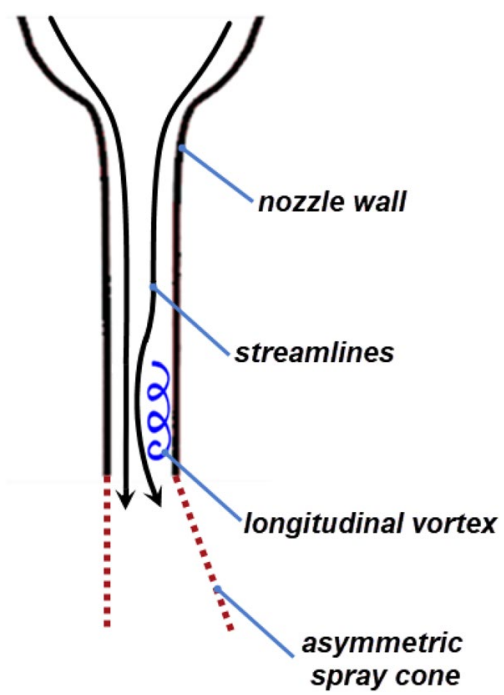

Fig. 13 Schematic illustrating the flow phenomena linking in-nozzle vorticity to spray-cone angle variation. An explicit cause/effect relationship is proposed to exist between the in-nozzle longitudinal vortex and the asymmetrically diverging spray flow

diffuse-light imaging only, yet are crucial for the performance evaluation of microfluidics devices.

Acknowledgements The work was performed at the Combustion Research Facility (CRF), Sandia National Laboratories, Livermore, CA. Sandia National Laboratories is a multi-mission laboratory managed and operated by National Technology and Engineering Solutions of Sandia, LLC., a wholly owned subsidiary of Honeywell International, Inc., for the U.S. Department of Energy's National Nuclear Security Administration under con-tract DE-NA0003525. Funding from the EU Horizon-2020 Marie Skłodowska-Curie Global Fellowships AHEAD (IK, Grant no. 794831) and UNIFIED (PK, Grant no. 748784) is acknowledged, which also supported the international visiting program of Ioannis K. Karathanassis and Phoevos Koukouvinis at the Sandia National Laboratories. Additional funding has been received by the UK's Engineering and Physical Sciences Research Council (EPSRC) through Grant EP/K020846/1.

Open Access This article is licensed under a Creative Commons Attribution 4.0 International License, which permits use, sharing, adaptation, distribution and reproduction in any medium or format, as long as you give appropriate credit to the original author(s) and the source, provide a link to the Creative Commons licence, and indicate if changes were made. The images or other third party material in this article are included in the article's Creative Commons licence, unless indicated otherwise in a credit line to the material. If material is not included in the article's Creative Commons licence and your intended use is not permitted by statutory regulation or exceeds the permitted use, you will need to obtain permission directly from the copyright holder. To view a copy of this licence, visit http://creativecommons.org/licenses/by/4.0/.

\section{References}

Aleiferis PG, Serras-Pereira J, Augoye A et al (2010) Effect of fuel temperature on in-nozzle cavitation and spray formation of liquid hydrocarbons and alcohols from a real-size optical injector for direct-injection spark-ignition engines. Int J Heat Mass Transf 53:4588-4606. https://doi.org/10.1016/j.ijheatmasstrans fer.2010.06.033

Andriotis A, Gavaises M, Arcoumanis C (2008) Vortex flow and cavitation in diesel injector nozzles. J Fluid Mech 610:195-215. https:// doi.org/10.1017/S0022112008002668

Arndt REA (2002) Cavitation in vortical flows. Annu Rev Fluid Mech $34: 143-175$

Baik S, Blanchard JP, Corradini ML (2003) Development of microdiesel injector nozzles via MEMS technology and effects on spray characteristics. At Sprays 13:443-474. https://doi.org/10.1615/ AtomizSpr.v13.i56.20

Batchelor GK (2000) An introduction to fluid dynamics. Cambridge University Press, Cambridge

Beloki Perurena J, Asma CO, Theunissen R, Chazot O (2009) Experimental investigation of liquid jet injection into Mach 6 hypersonic crossflow. Exp Fluids 46:403-417. https://doi.org/10.1007/s0034 8-008-0566-5

Ben-Yakar A, Hanson R (2002) Ultra-fast-framing schlieren system for studies of the time evolution of jets in supersonic crossflows. Exp Fluids 32:652-666. https://doi.org/10.1007/s00348-002-0405-z

Bloomberg NEF (2020) Electric vehicle outlook. https://about.bnef. com/electric-vehicle-outlook/. Accessed 29 June 2020

Blume M, Schwarz P, Rusche H et al (2019) 3D simulation of turbulent and cavitating flow for the analysis of primary breakup mechanisms in realistic diesel injection processes. At Sprays 29:861893. https://doi.org/10.1615/AtomizSpr.2020032492

Chaves H, Eberle A, Hofemeier P (2010) Micro-PIV for high velocity flows. In: 15th international symposium on applications of laser techniques to fluid mechanics. Lisbon, Portugal, July 5-8

Dumouchel C, Leboucher N, Lisiecki D (2013) Cavitation and primary atomization in real injectors at low injection pressure condition. Exp Fluids. https://doi.org/10.1007/s00348-013-1554-y

ECN (2020) Engine Combustion Network-Spray A\&B Target Conditions. https://ecn.sandia.gov/diesel-spray-combustion/target-condi tion/spray-ab/. Accessed 29 June 2020

Foeth EJ, Van Doorne CWH, Van Terwisga T, Wieneke B (2006) Time resolved PIV and flow visualization of 3D sheet cavitation. Exp Fluids 40:503-513. https://doi.org/10.1007/s00348-005-0082-9

Gavaises M, Andriotis A, Papoulias D et al (2009) Characterization of string cavitation in large-scale diesel nozzles with tapered holes. Phys Fluids 21:1-9. https://doi.org/10.1063/1.3140940

Ghiji M, Goldsworthy L, Brandner PA et al (2017) Analysis of diesel spray dynamics using a compressible Eulerian/VOF/LES model and microscopic shadowgraphy. Fuel 188:352-366. https://doi. org/10.1016/j.fuel.2016.10.041

Gopalan S, Katz J (2000) Flow structure and modeling issues in the closure region of attached cavitation. Phys Fluids 12:895-911. https://doi.org/10.1063/1.870344

Hayashi T, Suzuki M, Ikemoto M (2012) Visualization of internal flow and spray combustion with real size diesel nozzle. In: 12th triennial international conference on liquid atomization and spray systems, Heidelberg, Germany, September 2-6 
Hult J, Simmank P, Matlok S et al (2016) Interior flow and near-nozzle spray development in a marine-engine diesel fuel injector. Exp Fluids 57:49. https://doi.org/10.1007/s00348-016-2134-8

Karathanassis IK, Koukouvinis P, Kontolatis E et al (2018a) Highspeed visualization of vortical cavitation using synchrotron radiation. J Fluid Mech 838:148-164. https://doi.org/10.1017/ jfm.2017.885

Karathanassis IK, Trickett K, Koukouvinis P et al (2018b) Illustrating the effect of viscoelastic additives on cavitation and turbulence with X-ray imaging. Sci Rep 8:14968. https://doi.org/10.1038/ s41598-018-32996-w

Karathanassis IK, Koukouvinis P, Gavaises M (2020) Multiphase phenomena in diesel fuel injection systems. In: Singh A, Shukla P, Hwang J, Agarwal A (eds) Simulations and optical diagnostics for internal combustion engines current status and way forward. Springer, Singapore, pp 95-126

Kastengren A, Tilocco FZ, Duke D, Powell CF (2014) Time-resolved $\mathrm{X}$-ray radiography of diesel injectors from the engine combustion network. At Sprays 24:1-8

Kirsch V, Hermans M, Schönberger J et al (2019) Transparent highpressure nozzles for visualization of nozzle internal and external flow phenomena. Rev Sci Instrum 90:033702. https://doi. org/10.1063/1.5065658

Kolev N (2007) Thermodynamic and transport properties of diesel fuel. Springer, Berlin

Kolokotronis D, Hardalupas Y, Taylor AMKP, et al (2010) Experimental investigation of cavitation in gasoline injectors. SAE Tech Pap 2010-01-1500. https://doi.org/10.4271/2010-01-1500

Kouchi T, Goyne CP, Rockwell RD, McDaniel JC (2015) Focusingschlieren visualization in a dual-mode scramjet. Exp Fluids 56:114. https://doi.org/10.1007/s00348-015-2081-9

Koukouvinis P, Vidal-Roncero A, Rodriguez C et al (2020) High pressure/high temperature multiphase simulations of dodecane injection to nitrogen: application on ECN Spray-A. Fuel 275:117871. https://doi.org/10.1016/j.fuel.2020.117871

Kovesi P (1995) Image features from phase congruency. In: Technical Report 95/4. University of Western Australia

Laberteaux KR, Ceccio SL, Mastrocola VJ, Lowrance JL (1998) High speed digital imaging of cavitating vortices. Exp Fluids 24:489_ 498. https://doi.org/10.1007/s003480050198

Mamaikin D, Knorsch T, Rogler P, Wensing M (2020) Experimental investigation of flow field and string cavitation inside a transparent real-size GDI nozzle. Exp Fluids 61:1-15. https://doi.org/10.1007/ s00348-020-02982-y

Manin J, Pickett LM, Yasutomi K (2020) Stereoscopic high-speed microscopy to understand transient internal flow processes in high-pressure nozzles. Exp Therm Fluid Sci 114:110027. https:// doi.org/10.1016/j.expthermflusci.2019.110027

Mauger C, Méès L, Michard M et al (2012) Shadowgraph, schlieren and interferometry in a 2D cavitating channel flow. Exp Fluids 53:1895-1913. https://doi.org/10.1007/s00348-012-1404-3

Mishra C, Peles Y (2005) Flow visualization of cavitating flows through a rectangular slot micro-orifice ingrained in a microchannel. Phys Fluids 17:1-14. https://doi.org/10.1063/1.2132289

Mithun MG, Koukouvinis P, Gavaises M (2018) Numerical simulation of cavitation and atomization using a fully compressible threephase model. Phys Rev Fluids 3:1-27. https://doi.org/10.1103/ PhysRevFluids.3.064304

Moon S (2016) Novel insights into the dynamic structure of biodiesel and conventional fuel sprays from high-pressure diesel injectors. Energy 115:615-625. https://doi.org/10.1016/j.energ y.2016.09.062
Moon S, Huang W, Li Z, Wang J (2016) End-of-injection fuel dribble of multi-hole diesel injector: comprehensive investigation of phenomenon and discussion on control strategy. Appl Energy 179:7-16. https://doi.org/10.1016/j.apenergy.2016.06.116

Otsu N (1979) A threshold selection method from gray-level histograms. IEEE Trans Syst Man Cybern 20:62-66

Pastor JV, Payri R, Garcia-Oliver JM, Nerva JG (2012) Schlieren measurements of the ECN-spray a penetration under inert and reacting conditions. SAE Tech Pap 2012-01-0456. https://doi. org/10.4271/2012-01-0456

Payri F, Bermúdez V, Payri R, Salvador FJ (2004) The influence of cavitation on the internal flow and the spray characteristics in diesel injection nozzles. Fuel 83:419-431. https://doi.org/10.1016/j. fuel.2003.09.010

Phalnikar KA, Kumar R, Alvi FS (2008) Experiments on free and impinging supersonic microjets. Exp Fluids 44:819-830. https:// doi.org/10.1007/s00348-007-0438-4

Pickett LM, Genzale CL, Manin J (2015) Uncertainty quantification for liquid penetration of evaporating sprays at diesel-like conditions. At Sprays 25:425-452. https://doi.org/10.1615/AtomizSpr.20150 10618

Reid BA, Gavaises M, Mitroglou N et al (2014) On the formation of string cavitation inside fuel injectors. Exp Fluids. https://doi. org/10.1007/s00348-013-1662-8

Saric WS (1994) Goertler vortices. Annu Rev Fluid Mech 26:379-409

Schmidt DP, Corradini ML (2001) The internal flow of diesel fuel injector nozzles: a review. Int J Engine Res 2:1-22. https://doi. org/10.1243/1468087011545316

Settles GS (2001) Schlieren and shadowgraph techniques. Springer, Berlin, p 2001

Sun CL, Hsiao TH (2013) Quantitative analysis of microfluidic mixing using microscale schlieren technique. Microfluid Nanofluid 15:253-265. https://doi.org/10.1007/s10404-013-1148-2

Tan DJ, Edgington-Mitchell D, Honnery D (2015) Measurement of density in axisymmetric jets using a novel background-oriented schlieren (BOS) technique. Exp Fluids 56:1-11. https://doi. org/10.1007/s00348-015-2076-6

Vera-tudela W, Haefeli R, Barro C et al (2020) An experimental study of a very high-pressure diesel injector (up to 5000 bar) by means of optical diagnostics. Fuel 275:117933. https://doi.org/10.1016/j. fuel.2020.117933

Westlye FR, Penney K, Ivarsson A et al (2017) Diffuse back-illumination setup for high temporally resolved extinction imaging. Appl Opt 56:5028. https://doi.org/10.1364/ao.56.005028

Wilhelm D, Hrtel C, Kleiser L (2003) Computational analysis of the two-dimensionalthree-dimensional transition in forward-facing step flow. J Fluid Mech 489:1-27. https://doi.org/10.1017/S0022 112003004440

Yasutomi K, Hwang J et al (2020) Transient internal nozzle flow in transparent multi-hole diesel injector. SAE Tech Pap 2020-01-08. https://doi.org/10.4271/2020-01-0830.Abstract

Yick K-Y, Stocker R, Peacock T (2006) Microscale synthetic schlieren. Exp Fluids 42:41-48. https://doi.org/10.1007/s00348-006-0217-7

Publisher's Note Springer Nature remains neutral with regard to jurisdictional claims in published maps and institutional affiliations. 